\title{
SUM THINGS ARE NOT WHAT THEY SEEM: PROBLEMS WITH THE INTERPRETATION AND ANALYSIS OF RADIOCARBON-DATE
}

\section{PROXIES}

\section{A PREPRINT}

\author{
W. Christopher Carleton \\ Extreme Events Research Group \\ Max Planck Institute for Chemical Ecology \\ 07745 Jena, Germany \\ wcarleton@ice.mpg.de
}

\author{
Huw S. Groucutt \\ Extreme Events Research Group \\ Max Planck Institute for Chemical Ecology \\ 07745 Jena, Germany \\ hgroucutt@ice.mpg.de
}

November 11, 2019

\begin{abstract}
Radiocarbon-date proxies are widely used in studies exploring long-term variation in human and environmental phenomena. Examined phenomena include, for example, variation in past human population levels and climate-change-driven sea level fluctuations. These processes are thought to have affected the amount of organic carbon deposited into the archaeological and/or palaeoenvironmental record at a given time. Time-series representing through-time fluctuations in the frequency of dated radiocarbon samples are, therefore, often used as proxies for such processes. However, there are important problems with radiocarbon-date proxies that have so far gone underappreciated in the scientific literature. The primary problem is that the proxies are easily misinterpreted, and this has serious implications for downstream analyses. Here we report the results of a two-part study. In the first part, we investigated the most accurate interpretation of radiocarbon-date proxies produced with each of the two established methods, widely-used summed probability density functions and a new kernel density estimation approach. In the second part, we performed a simulated regression experiment to determine whether the proxies could be used to quantitatively investigate the processes they are often thought to represent. Our analyses unfortunately reveal that the proxies do not reflect what they are generally thought to-i.e., through-time variation in processes correlated with radiocarbon sample frequency. Rather, they represent a combination of through-time variation in sample frequency and chronological uncertainty. More importantly, though, our regression experiment demonstrated that
\end{abstract}


the proxies can produce very misleading results. While the proxies may be useful under certain conditions for addressing certain kinds of research questions, they are not generally suitable as representations of through-time processes. A major implication of this finding is that a significant number of high-profile published studies may be reporting false results based on misinterpretations of core data. Another major implication is that the proxies should be avoided in future research when the goal is to understand through-time variation in a given process.

Keywords Summed Radiocarbon Dates - Summed Probability Density Functions - Archaeological Proxies · Palaeoenvironmental Proxies $\cdot$ Radiocarbon $\cdot$ Palaeoclimatology $\cdot$ Sea Level Changes

\section{Introduction}

Radiocarbon-date proxies have become popular in archaeological and palaeoenvironmental research. Often referred to as summed probability distribution/density functions (SPDFs), the proxies are often thought to represent throughtime fluctuations in a target process that affected the amount of organically derived radiocarbon deposited into the archaeological and palaeoenvironmental records. The target processes include, for example, population levels in archaeological research (e.g., d'Alpoim Guedes et al., 2016; Edinborough et al., 2017; Riede, 2009; Shennan, 2009) or past climate changes in palaeoenvironmental research (e.g., Bleicher, 2013; Thorndycraft and Benito, 2006; Turney and Brown, 2007). Interest in using the proxies has also led to methodological research resulting in the identification of important biases and the development of new techniques (e.g., Brown, 2015; Chiverrell et al., 2011; Crema et al., 2017; Kerr and McCormick, 2014; Surovell and Brantingham, 2007; Williams, 2012), including a new kernel density estimation (KDE) method for constructing them (Ramsey, 2017). Widespread adoption of the proxies is having a significant impact on our understanding of both human-environment dynamics and climatic phenomena. However, crucial problems with the proxies have been overlooked, particularly regarding their interpretation and use in downstream statistical analyses. Here we report the results of a two-part study. In the first part, we carefully explored the mathematical details of SPDF and KDE models to uncover their most accurate interpretations. Then, in the second part, we used simulated data and regression analyses to evaluate whether the proxies can be used as response variables in place of their intended target process-i.e., through-time variations in the number of radiocarbon-dated events.

\section{Background}

The idea that radiocarbon dates could be used for something other than chronological control first appeared in a 1969 paper by Mebus Geyh. The paper reports a study of Holocene sea level changes on the North Sea coast with the help of "statistischen Auswertung von ${ }^{14} \mathrm{C}$-Daten" ("statistical evaluation of ${ }^{14} \mathrm{C}$ data")(Geyh, 1969). In the paper, Geyh argued that the amount of radiocarbon in different layers of sediment from around the North Sea could be used as a proxy for past through-time fluctuations in sea level. His argument was based on the relationship between marine ingression, wetland formation, peat deposits, and carbon preservation. When sea levels rise, the argument went, coastal 
areas experience an increase in wetland formation. This increase in turn leads to the formation of peat and, as a consequence, increased carbon preservation in sediments. Thus, Geyh observed, sediment cores will contain more carbon samples in portions that formed during periods of high sea level. Using this logic, he proposed a method for turning a set of radiocarbon dates into a proxy for past sea-level changes. The approach entailed approximating the Gaussian radiocarbon-date distributions with step-functions and then summing those functions (see Fig. 1). Using this approach, Geyh created several sea-level proxies based on radiocarbon samples from coastal sediment cores around the North Sea. Each proxy corresponded to a sub-region within the wider study area. He then compared the sub-regional proxies and identified peaks in the proxies that appeared to be contemporaneous. These coeval peaks, he claimed, demonstrated sea-level change in the North Sea was regionally synchronous. Geyh then went on to use the technique in several more studies of Holocene climate change (e.g., Geyh, 1971, 1980).

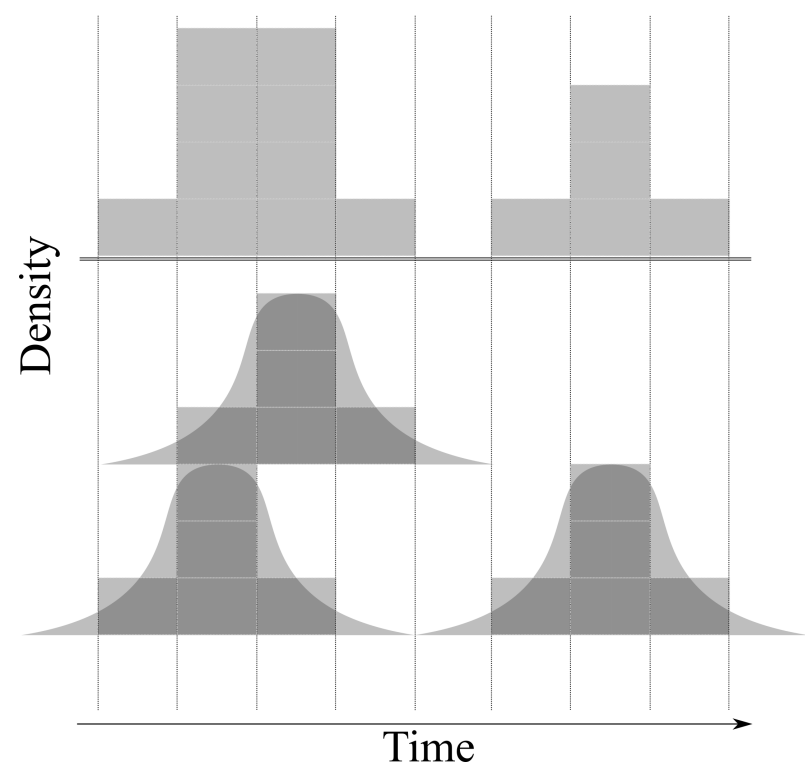

Figure 1: Step function summing technique developed by Geyh 1969. Each radiocarbon-date density (represented by smooth Gaussian distributions in this image) is first approximated by a step function (represented here by blocks). Then, the height of each step function is summed for each interval of time to produce the summed radiocarbon-date proxy, which is represented in this image by the blocky histogram-like figure above the double-line.

Independently, it seems, a similar method was introduced to archaeology in 1987 by John Rick in an article titled "Dates as Data: An Examination of the Peruvian Preceramic Radiocarbon Record" (Rick, 1987). Rick argued that radiocarbon dates could be used as a proxy for past population levels because humans have a sort of archaeological carbon footprint. He based this argument on the observation that people use and produce carbon that ultimately ends up in the archaeological record. Every piece of firewood or timber, or burned seed or bone, is at some point deposited into the sedimentary record - this includes, of course, human remains. Consequently, according to Rick, more people means more organic carbon deposits. Thus, he averred, at large enough spatial and temporal scales the number of people alive at a given time should correspond to the amount of organic carbon deposited into the archaeological record from that time. He applied the same logic to spatial variation-more people in a given region means more organic carbon deposits in that region. Then, in his view, as archaeologists recover and date the deposited carbon, the number of radiocarbon 
dates from a given period or region can be expected to correlate with the population levels during that period and/or in that region. As a case study, Rick used this argument to interpret regional and temporal variation in radiocarbon dates from Peruvian archaeological sites dated to between 20,000 and 3000 B.P. He binned the radiocarbon dates into regions and histograms with temporal intervals of 200- and 600-years. Then, based on the temporal patterns in the histograms and comparisons between regions, he posited several hypotheses about human population expansion, contraction, and mobility throughout most of the last 20,000 years in Peru.

Since their inception, radiocarbon-date proxies have become wildly popular. A Web-of-Science search for the topic "summed radiocarbon" in archaeological and interdisciplinary palaeoenvironmental science journals returned over 100 articles published since 2010 and a similar Google Scholar search returned over 400 articles. In palaeoenvironmental research the approach has been widely adopted, and the proxies have been used to study a variety of target phenomena including past sea level changes, fire-regime surges, and climatic changes (e.g., Bleicher, 2013; Mooney et al., 2011; Pierce et al., 2004; Thorndycraft and Benito, 2006). Archaeologists have been particularly enthusiastic, routinely using radiocarbon dates as a proxy for past population levels (e.g., Armit et al., 2013; Bamforth and Grund, 2012; Collard et al., 2010; Colledge et al., 2019; Faulkner, 2011; Gamble et al., 2005; Leipe et al., 2019; Lepofsky et al., 2005; Prentiss et al., 2014; Schulting, 2010; Shennan, 2013; Steele, 2010; Turney and Brown, 2007). Recent applications have, of course, improved since Geyh and Rick's original papers. The current, most popular method of producing the proxies (the SPDF) produces much higher temporal resolutions than the approaches used by Geyh and Rick—on the order of 1-5 years instead of centennial scales - and the proxies are typically based on massive radiocarbon date databases comprising hundreds or thousands of dates (e.g., Manning and Timpson, 2014; Shennan, 2013; Shennan et al., 2013). Radiocarbon dating techniques have also improved significantly, resulting in substantial gains in precision and certainty of individual dates (Devièse et al., 2017; Ramsey, 2008). And, many scholars now use Bayesian methods (Buck et al., 1996, 1992; Ramsey, 2009) to constrain radiocarbon-dating uncertainties with prior knowledge about stratigraphic relationships among radiocarbon samples.

Beginning with Geyh 1969 and Rick 1987, however, scholars have also been well aware of several key sources of bias affecting the proxies (e.g., Bleicher, 2013; Brown, 2015; Crema et al., 2017; Kerr and McCormick, 2014; Manning and Timpson, 2014; Williams, 2012). One involves the quality of the dates. Geyh was especially concerned about radiocarbon leaching from younger into older deposits, which would make the older deposits appear to be too young (Geyh, 1969, 1971). Quality can also be affected by "chronometric hygiene" (Brown, 2015), which refers to a number of criteria used to assess the quality of the samples. These criteria include clear sedimentological associations with the target process, and the use of samples from short-lived organisms, such as seeds, to overcome the "old wood problem"a problem whereby the sedimentary layer containing a given sample is actually much younger than the carbon sample itself (Schiffer, 1986). Another source of bias involves sampling, discussed by both Geyh 1969 and Rick 1987 in their initial papers and more recently by many others (e.g., Crema et al., 2017; Williams, 2012). This bias stems from the fact that not all regions and/or time periods have been studied with the same intensity, which can lead to spurious differences between regions and time periods. A third source of bias involves preservation. Taphonomic processes 
determine how much carbon survives to the present, so taphonomic processes that lead to differential preservation among organic carbon sources, time-periods, and regions must be considered when using radiocarbon dates as data (Brown, 2015; Surovell and Brantingham, 2007; Williams, 2012). A fourth source of bias involves spurious features introduced into the proxies by radiocarbon calibration. Radiocarbon dates have to be calibrated so that they can be mapped onto calendar time (Taylor et al., 2014). The process involves a time-series of securely-dated carbon samples that relates radiocarbon years to calendar years (Reimer et al., 2013). These calibration curves contain their own uncertainties and features that have nothing to do with the number of radiocarbon samples but can nonetheless affect the shape of radiocarbon-date proxies. Geyh identified and described this problem in 1980 with regard to Suess wiggles (Geyh, 1980)—decadal-to-centennial scale fluctuations in calibration curves—and more recent studies have confirmed that calibration introduces artifacts into the proxies (Brown, 2015; Kerr and McCormick, 2014). Lastly, there is a bias specific to archaeological use of radiocarbon proxies involving culture. Rick noted that different cultural, technological, and economic conditions could lead to differences in the amount of carbon deposited per "unit of occupation" (Rick, 1987), an abstract term he used to describe both the population level and activity intensity in a given society.

The widespread recognition of these biases has led to a number of compensatory, bias-correcting suggestions (see Crema et al., 2017; Williams, 2012). To address issues of sample quality, several scholars have advocated carefully selecting radiocarbon dates based on the perceived reliability of the dates and quality of the dated material—including the use of short-lived samples to avoid the old-wood problem (e.g., Collard et al., 2010; Ebert et al., 2017; Hoggarth et al., 2016; Shennan, 2013). To overcome sampling issues, most scholars argue that large databases of dates are required (e.g., Shennan, 2013; Williams, 2012), although little certainty exists about what constitutes a 'good' sample size in this setting. And, with regard to taphonomic bias, Surovell et al. 2009 suggested that independently dated tephra-based proxies for taphonomic loss could be used to correct the radiocarbon-date proxies.

In addition to these recommendations, recent research has also led to relevant methodological advances. Shennan et al. 2013, for instance, introduced a method for testing the statistical significance of fluctuations in the proxies. The authors were attempting to address questions about the relationship between climate change and population levels in Western Europe before, during, and after the arrival of farming in a given region. To do so, they created a radiocarbon-date proxy for demographic change using a large database of over 7000 dates. They reasoned, however, that one or more of the biases noted above may have produced misleading through-time fluctuations in the proxy. To compensate, they used a simulation-based approach in which they generated a set of random dates from an exponential model of population change through-time. The exponential growth model served as a baseline-i.e., a null hypothesis-against which they compared the actual radiocarbon-date proxy. They found that the proxy did contain significant deviations from the null, leading them to conclude that uninterrupted exponential growth could not account for the observed changes in the proxy and that environmental fluctuations were a plausible alternative.

Another recent development was the introduction of spatial permutation tests to compensate for spatial variations in sampling intensity (Crema et al., 2017). Similar to Rick's original study, most spatially-oriented analyses of radiocarbondate proxies published so far have involved binning the relevant radiocarbon dates into discrete regions (e.g., Gayo 
et al., 2015; Pierce et al., 2004; Turney and Brown, 2007). More recently, though, some scholars have attempted to handle the spatial dimension of the radiocarbon-date proxies in a continuous manner (e.g., Collard et al., 2010; García Puchol et al., 2018; Manning and Timpson, 2014). The problem with doing so, as explained by (Crema et al., 2017), is that continuous approaches can magnify bias introduced by spatially heterogeneous sampling intensity-i.e., spatially localized features in the data reflect heterogeneous sampling density rather than real differences in the time-space distribution of dated radiocarbon samples. To combat this source of bias, Crema et al. 2017 proposed a permutation test. The method involves creating local summed radiocarbon-date proxies within a region and then shuffling the locations of the proxies randomly. Each time the locations are shuffled, a set of rates of change in each local summed proxy are estimated. This set of rates gives rise to a distribution of rates that can be used for hypothesis testing. If the true proxy for a given location has a rate of change that looks extreme relative to the permutation distribution, that local change is considered significant. The test amounts to comparing local rates of change within a given region to the global distribution of change rates for the whole region.

The last, perhaps most significant, development is a proposed change in the fundamental way radiocarbon-date proxies are produced. The new method, developed by Christopher Ramsey, uses kernel density estimation (KDE) to create a proxy (Ramsey, 2017). KDE is a commonly used non-parametric method for estimating the continuous probability density of a random variable given a finite set of realizations of that variable (Silverman, 1986). In the case of radiocarbon-date proxies, the realizations are an observed set of radiocarbon dates while the density is the temporal distribution of those dates. The kernel is essentially a moving window that computes a weighted-sum of the number of radiocarbon-dated events that occurred in a given time. It assigns weights based on the temporal distance from the centre of the kernel to the date of a given sample. The closer a given date is to the centre of the kernel, the more it contributes to the level of the proxy at the time corresponding to the kernel's centre. The KDE model also incorporates the chronological uncertainty of the radiocarbon dates and it can be nested within a Bayesian calibration model so that it reflects prior information about the relative sedimentological relationships among the radiocarbon samples.

\section{Reasons for Skepticism}

Despite all of the recent methodological research there are at least two reasons to be skeptical of radiocarbon-date proxies. Chiefly, the exact interpretation of the proxies is unclear. In theory, at least, the arguments laid out by Geyh 1969 and Rick 1987 stand to reason. Given sufficiently large samples and assuming minimal biases, the processes that produced radiocarbon samples should correspond to the number of samples recovered and, therefore, the number of radiocarbon dates. This goes for both natural and anthropogenic processes. Individual radiocarbon-date densities, however, represent chronological uncertainty. They do not represent duration or through-time variation in a process that produces radiocarbon samples. The chronological uncertainty represented by individual radiocarbon-date densities is subsequently included in radiocarbon-date proxies. This feature has not been sufficiently interrogated, in our view, leaving open a crucial question about the most accurate interpretation of the proxies. 
The other reason for continued skepticism is that the proxies have not been sufficiently evaluated with respect to their most promising potential use, namely regression analyses. Thus far, most scholars using radiocarbon-date proxies have treated them as representations of a target process that varies through time. A key motivation for creating such a proxy is to explain variation in its target process by comparing the proxy to one or more covariates that could serve as potential causal factors. This motivation naturally gives rise to quantitative analyses, like regression models, as pointed out by Crema et al. (Crema et al., 2017). It is, therefore, important to ask whether the proxies perform adequately in a quantitative regression context. So far as we are aware there have been no attempts to systematically answer this question.

Together, these reasons for skepticism suggest there may be serious problems with the use of radiocarbon-date proxies. The proxies may not represent what they are often thought to represent and they may be unsuitable for downstream statistical analyses, such as regression. With these problems in mind, we conducted a two-part study. In the first part, we carefully explored the mathematical definitions of the two established proxies, SPDF and the new KDE model. The primary aim of this part of our study was to determine the most accurate interpretation of through-time fluctuations in both proxies. In the second part, we conducted a simple simulated regression experiment. For this experiment, we simulated a count-based time-series of calendar events using a linear model and a single covariate. The model included a regression coefficient that related the simulated covariate to the mean of a Poisson process, which ultimately produced the count-based time-series. Next, we converted the series of counts-i.e., number of events per year-into a set of calendar dates. We then used the well-known radiocarbon calibration program, OxCal (Ramsey, 2009), to simulate radiocarbon assays and corresponding calibrated-date densities from the simulated "true" calendar dates. Lastly, we attempted to estimate the known relationship between the simulated count-based time-series (i.e., the target process) and the covariate by using the radiocarbon-date proxies in place of the target process. We reasoned that if the radiocarbon-date proxies really corresponded to the target process, the covariate would appear statistically significant in our regression analyses.

\section{Interpreting Radiocarbon Date Proxies}

In this section, we explore the mathematics behind radiocarbon-date proxies in an effort to reveal their most accurate interpretation. To provide some context, imagine a hypothetical research scenario in which a large database of radiocarbon dates has been amassed. Suppose that the individual dates represent either peat accumulation representative of marine ingression, or some archaeological find that correlates with past population levels-say, individual domestic structures. Further suppose that there is no need for a complex Bayesian calibration model because the individual radiocarbon dates in the imaginary database are not related stratigraphically. Also assume that the dated samples are reliable and that all of the aforementioned biases have been compensated for-i.e., there is no effect of taphonomic loss and the number of radiocarbon samples actually does represent the target process in question. These simplifications aid only in the exploration of the simplest kinds of radiocarbon proxies and more complex scenarios could be considered in the same manner. Put another way, the simplifying assumptions can be employed for clarity without loss of generality. 


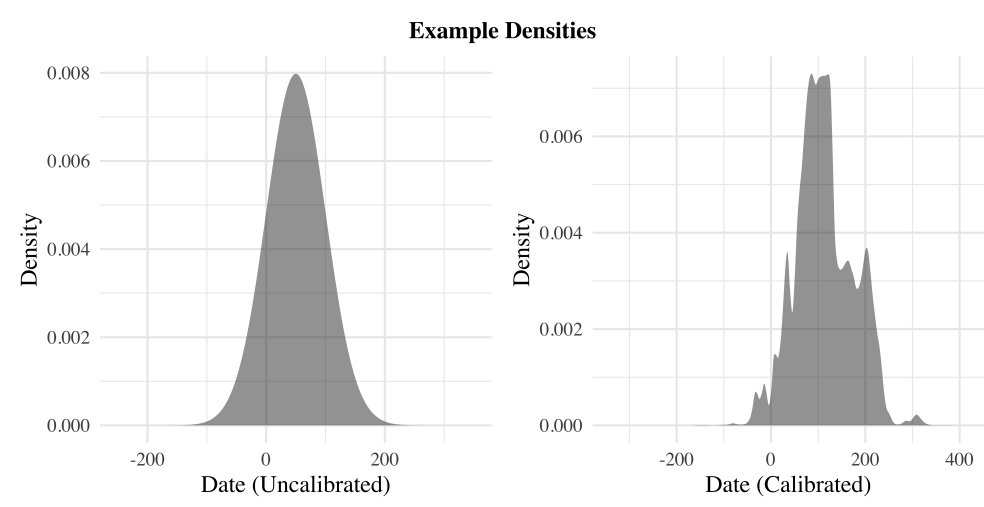

Figure 2: Example radiocarbon date densities. These densities represent an uncalibrated radiocarbon date of 1900 BP $(50 \mathrm{CE})$ with an error of \pm 50 years. The plot on the left shows the uncalibrated distribution and the plot on the right shows the calibrated distribution produced by OxCal (Ramsey, 2009). The height of each curve for a given year indicates the probability that the sample dates to the relevant year compared to other years.

\subsection{SPDF}

First, we explored the SPDF. Let $p(\tau)$ be a single radiocarbon date density where $\tau$ refers to a single year in the domain (x-axis) of the density. The level of the density-i.e., height with respect to the y-axis—at any given $\tau$ is proportional to the relative probability that the sample dates to $\tau$ compared to other times (see Fig. 2)(Buck et al., 1996; Ramsey, 2009). Next, let $p_{n}(\tau)$ be a radiocarbon date density, $n$, from a set of $N$ such densities. It is important to note that since the domain (time) is common to all densities, $\tau$ in any $p_{n}(\tau)$ refers to a particular year in a given interval of years $\left[\tau_{a}, \tau_{b}\right]$. Then, the sum of the $N$ densities for a given $\tau \in\left[\tau_{a}, \tau_{b}\right]$, denoted $S(\tau)$ is

$$
S(\tau)=\sum_{n}^{N} p_{n}(\tau) .
$$

That $p_{n}(\tau)$ is proportional to the probability that event $n$ occurred in year $\tau$ has implications for the interpretation of the summed function in eq.4.1. To see the implication clearly, we can expand the summation in eq.4.1 as follows:

$$
S(\tau)=p_{1}(\tau)+p_{2}(\tau)+\ldots+p_{N}(\tau) .
$$

Since the terms in the sum are proportional to probabilities, the sum is a quantity proportional to the sum of the individual probabilities. We can, therefore, treat them as equivalent to probabilities and look to standard theory for the correct interpretation of $S(\tau)$.

Given standard probability theory (Kolmogorov Probability Theory), there are two potential interpretations of $S(\tau)$ (the SPDF). The difference between them depends on the relationship between the individual events. Both interpretations involve the probability of the union of the individual events, which is calculated using the sum rule (Blitzstein and 
Hwang, 2015). The sum rule, as the name suggests, describes the situations in which probabilities can be summed (as in eq. 4.2) and the interpretations of the resulting sums.

For the first interpretation, we assume that the events are mutually exclusive, meaning that they cannot co-occur. Consider, for example, events $A$ and $B$, with corresponding probabilities, $P(A)$ and $P(B)$. When the events are mutually exclusive-i.e., only one or the other can happen, not both—the sum rule is as follows (Blitzstein and Hwang, 2015),

$$
P(A \cup B)=P(A)+P(B) .
$$

With respect to a pair of radiocarbon dates, mutual exclusivity would mean that the two events in question could not have happened on the same date. Or, more formally, given two events, $p_{1}\left(\tau_{i}\right)$ and $p_{2}\left(\tau_{j}\right)$,

$$
P\left(p_{1}\left(\tau_{i}\right) \cap p_{2}\left(\tau_{j}\right)\right)=0: i=j,
$$

where $\tau_{i}$ and $\tau_{j}$ each refer to a date on a common time-axis.

Substituting into eq. 4.3 two mutually exclusive radiocarbon-dated events- $p_{i}(\tau)$ and $p_{j}(\tau)$-the right-hand side of eq. 4.3 is equivalent to the SPDF in eq.4.2:

$$
P\left(p_{i}(\tau) \cup p_{j}(\tau)\right)=S(\tau)=p_{i}(\tau)+p_{j}(\tau) \mid i \neq j .
$$

The interpretation in this case is straightforward and meaningful. It is the probability that at least one of the individual events occurred (Blitzstein and Hwang, 2015). In archaeological terms, it would be the probability that at least one of our imaginary radiocarbon-dated domestic buildings dates to a given year. In the strictest sense, of course, it would be the probability that the object used to date a given layer of archaeological or palaeoenvironmental sediment—e.g., a bone, seed, or tree-stopped absorbing radiocarbon from the environment at a given time. Nonetheless, assuming the events in question are mutually exclusive, the level of a given SPDF at a given time has a meaningful interpretation. It is the probability that at least one of the events dates to that time. This is essentially the interpretation described in the reference manual for OxCal under the "sum function" entry (https://c14.arch.ox.ac.uk/oxcalhelp/hlp_ commands.html, accessed 2019-11-01).

If, however, the individual events are not mutually exclusive, the probability of the union must be calculated in a slightly different way and this yields the second interpretation for $S(\tau)$. According to the sum rule, the probability of the union of non-mutually exclusive events is given by 


$$
P(A \cup B)=P(A)+P(B)-P(A \cap B),
$$

where the last term- $P(A \cap B)$-is the probability of the intersection of the events (Blitzstein and Hwang, 2015). This intersection refers to the portion of the probability space in which both events occur. In scientific terms, it would be the probability that both events (e.g., construction dates of domestic structures, or sediment deposited by marine ingression) date to the same time. But, the summation in eq.4.1 contains no such term. Standard SPDFs are calculated without considering the probability of the intersection of events. Consequently, in the case of non-mutually-exclusive events, $S(\tau)$ is not proportional to the probability of the union of the individual events. Instead it must be greater by some quantity proportional to the probability of the intersection of the events in question. Again using two hypothetical radiocarbon-dated events, for example, eq. 4.6 becomes,

$$
P\left(p_{i}(\tau) \cup p_{j}(\tau)\right)=p_{i}(\tau)+p_{j}(\tau)-P\left(p_{i}(\tau) \cap p_{j}(\tau)\right) \mid i \neq j
$$

Thus, considering

$$
S(\tau)=p_{i}(\tau)+p_{j}(\tau) \mid i \neq j
$$

it is clear that

$$
S(\tau)>P\left(p_{i}(\tau) \cup p_{j}(\tau)\right) \mid i \neq j
$$

As a result, when the events in a given sample are not mutually exclusive, an SPDF of the events has no meaningful interpretation with respect to its level at any given time. Its level, $S(\tau)$, is only greater than a quantity proportional to the probability that at least one of them occurred at a given time (eq. 4.9).

This is a serious problem for studies involving SPDFs published so far. There is no reason to suspect that, in general, radiocarbon-dated events in large databases are all mutually exclusive. In fact, it seems very unlikely for that to be true. If it were, it would mean that none of the events in question could have co-occurred-a very strong assertion that would require significant evidence. While in the strictest sense it might also be unlikely that any two events were precisely simultaneous - the probability of simultaneity diminishing to zero as temporal precision increases—-that fact alone does not justify assuming a priori that any or all events were mutually-exclusive. Consequently, most if not all published SPDFs should be interpreted as indicating through-time variations in some quantity greater than another quantity 
proportional to the probability that at least one of the events in the relevant data set occurred at a given time. If that interpretation seems bewildering, then the point is clear. Worse still, though, the magnitude of the difference cannot be easily known. Thus, SPDFs calculated in the standard ways should be interpreted cautiously-much more so than they have been-and perhaps viewed with some measure of suspicion. Even a simple visual assessment would give the wrong impression if the viewer thought the variation in the proxy was plainly indicative of through-time fluctuations in the temporal density of events represented in a given database of radiocarbon dates.

Theoretically, the problem could be corrected by including the intersection denoted by the right-most terms in eqs.4.6 and 4.7, but calculating the probability of that intersection is generally going to be very difficult. For two events, the intersection, $P\left(p_{i}(\tau) \cap p_{j}(\tau)\right)$, is just the product of the individual probabilities-assuming the relevant events are independent, that is (Blitzstein and Hwang, 2015). Thus, the SPDF for two independent non-mutually exclusive events could be recalculated rather straightforwardly, resulting in a meaningful interpretation. For more than two events, however, the calculation becomes increasingly complicated as the number of events increases. The inclusion-exclusion principle - a formula accounting for all combinations of possible event intersections - is required to properly calculate the final probability of the union (Blitzstein and Hwang, 2015). This is possible given the right code and modern computers, but for databases involving hundreds to thousands of dates it would entail evaluating probabilities for upwards of $10^{30}$ to $10^{300}$ intersections. Even though these would be simple calculations individually, storing so many values would quickly exhaust the memory limits of most personal devices and the total computation time would be prohibitive unless high-performance computing clusters were used. In terms of memory, for example, assuming 1000 dates, merely generating all combinations of 3 dates $-C\left(\begin{array}{c}1000 \\ 3\end{array}\right)$-required 93 seconds and $2 \mathrm{~Gb}$ of memory on a MacBook Pro 2018 (in R). To be clear, these estimates reflect no calculations, only the time and space required to group the combinations of 3 elements from a sample of 1000 elements. Finding all the necessary combinations of 1000 dates would entail grouping all combinations of 1 date, 2 dates, 3 dates, 4 dates, and so on up to 1000 dates. We reached the built-in memory limits for $\mathrm{R}$ on a high-end machine at combinations of 4 dates. The situation is considerably more complicated if the events in question are not independent. In that case, the intersection is no longer the simple product of the individual probabilities. It is hard to imagine that independence among radiocarbon-dated events would be the rule in a real database. Some may be independent while others may not. So, the probability relationships among each and every date would have to be determined and included in the calculation of the intersection, potentially greatly increasing the analytical complexity and computational requirements.

Even if this hypothetical correction were applied, though, another problem would be lurking. The problem is that the level of an SPDF does not relate clearly to the number of events at any given time. To reiterate, given an SPDF that corresponds directly to the probability of the union of events, the interpretation of the level of the SPDF at a given time is the probability that at least one event occurred at that time. However, that probability has no necessary connection to the probability that any specific number of events occurred. The SPDF at a given time may be high because one of the events in the sample had a very high probability of occurring at the given time, or because several events each had some lower probability. This may be less a problem for large databases wherein individual events have less impact on the 
large-scale features of the SPDF. But, it is still a critical interpretive problem that should shake one's confidence in the SPDF as a useful proxy for through-time changes in the number of events in a given sample.

A proponent might argue that the probability that at least one event occurred at a given time really does correlate to the probability that more than one occurred at that time. While debatable, the argument could be taken for granted and a final, fatal problem would linger. The problem is that while most uses so far have involved interpreting SPDFs as a rough proxy for through-time changes in the number of events in a given database, the changes in the level of the proxy are also a function of chronological uncertainty. In fact, the whole proxy represents a summary of chronological uncertainty about the temporal location of the events in the database. This is clear from the definition of radiocarbon date—and calibrated radiocarbon-date—densities. As noted earlier, given a single radiocarbon-date density, $p(\tau)$, the level of $p(\cdot)$ at a given $\tau$ corresponds to the probability that the event in question dates to that time relative to another time. It does not reflect any through-time process. So, a sum of such densities, as in eqs. 4.1 and 4.2 must also be interpreted as representing changes in relative probabilities that reflect chronological uncertainty, rather than as a process representing through-time variation in the underlying number of events. Variation from one time to the next in the level of the SPDF indicates only a change in the level of chronological certainty that one or more of the underlying radiocarbon events dates to the relevant time. There need not be any change in the number of underlying events for there to be fluctuations in level of an SPDF. As a result, fluctuations in the proxy with respect to time cannot be interpreted as reflections of fluctuations in the number of dated events or, by extension, a processes thought to have produced through-time variation in the number of dated events.

\subsection{KDE Model}

The KDE model recently proposed by Christopher Ramsey (Ramsey, 2017) is constructed in a very different way than a standard SPDF. Rather than summing radiocarbon-date densities at a given temporal resolution, the KDE model is based on estimating the temporal density of of radiocarbon samples. As a result, the KDE model has a different interpretation than an SPDF.

Kernel density estimation is a method for approximating an unobservable continuous distribution from a finite observed sample [KDE]. In the context of radiocarbon-date proxies, the samples are a database of radiocarbon dates and the continuous distribution refers to the distribution of the relevant events in time. If there was no chronological uncertainty, the continuous density at any given time, $\tau$, of a set of events, $\mathbf{t}=\left\{t_{1}, t_{2}, \ldots, t_{n}\right\}$, could be approximated by a simple kernel density estimator,

$$
\hat{f}(\tau)=\frac{1}{h n} \sum_{i=1}^{n} K\left(\frac{\tau-t_{i}}{h}\right),
$$

where $h$ refers to the bandwidth of the kernel (Ramsey, 2017). The kernel can be thought of as a kind of moving window and the bandwidth as the width of the window. As the window slides along the time-axis, it estimates the level of the 
density function at a time, $\tau$, corresponding to its centre. The density at the centre is a weighted average of the number of events within the window, with weights inversely correlated to distance from the centre to each event. But, unlike a moving window, the kernel density is continuous. So, instead of hard boundaries like the ones defining window edges, the kernel applies a smooth distance decay function. As a result, the weights applied to each event in the database are a function of distance and all of the events are included in the sum. The further away in time an observed event is from the centre of the kernel, the less it counts toward the density at $\tau$. The bandwidth parameter helps to determine how rapidly the assigned weights decay with distance — a wider bandwidth would give more weight to observations further away in time.

In order to account for chronological uncertainty in the temporal locations of radiocarbon-dated events, Ramsey's 2017 KDE approach involves a simulation. Essentially, the simulation has three main steps. First, a sample of potential dates, $\hat{\mathbf{t}}=\left\{\hat{t}_{1}, \hat{t}_{2}, \ldots, \hat{t}_{n}\right\}$, is randomly drawn from the set of radiocarbon-date densities. Importantly, the probability of a given date being drawn from a given density is proportional to the level of the density corresponding to that date. Then, a bandwidth modifier, $g$, is randomly drawn from a theoretical prior uniform distribution, $g \sim U(0,1)$, and the following kernel density function is used to estimate the density of the sample, $\hat{\mathbf{t}}$,

$$
\hat{f_{h_{S}}}(\tau)=\frac{1}{g h_{S} n} \sum_{i=1}^{n} K\left(\frac{\tau-\hat{t_{i}}}{g h_{S}}\right) .
$$

where $h_{S}$ refers to the commonly used Silverman bandwidth (Silverman, 1986). Lastly, the likelihood of that density estimate and corresponding bandwidth modifier, $g$, is calculated. This process is repeated a large number of times to obtain a posterior density for $g$ and a likelihood-weighted average KDE model. The whole simulation is nested within the Markov-Chain Monte Carlo (MCMC) simulation used in OxCal (Ramsey, 2009) to calibrate radiocarbon dates. This means that prior information about stratigraphic relationships can be included and will be respected by the KDE model. OxCal will also output an ensemble of KDE models, each of which represents a KDE calculated with a slightly different bandwidth because each will have used a slightly different $g$ parameter. The ensemble can be used to provide a kind of uncertainty envelope for the primary, likelihood-weighted average KDE model.

A precise interpretation of the KDE model can be determined by looking at the way it accounts for chronological uncertainty. As we explained, each iteration of the simulation used to produce a KDE model involves randomly drawing a set of probable dates for a given set of radiocarbon samples. Let $\hat{\mathbf{t}}=\left\{\hat{t}_{1}, \hat{t}_{2}, \ldots, \hat{t}_{n}\right\}$ be the set of probable dates. Now imagine focusing in on a specific time $(\tau)$ with a kernel that has a given bandwidth $\left(g h_{S}\right)$. The level of the kernel function, $K$, at $\tau$ is primarily determined by the temporal distance between $\tau$ and a given set of dates, $\tau_{\Delta_{i}}=\tau-\hat{t}_{i}$. Treating the ratio outside the sum in eq. 4.11 as a proportionality constant and leaving it out for the moment means we can rewrite the equation more simply as follows, 


$$
\hat{f}(\tau) \propto \sum_{i=1}^{n} K\left(\frac{\tau_{\Delta_{i}}}{g h_{S}}\right) .
$$

From this equation, it is clear that variation at $\tau$ between potential KDE models arises from repeatedly re-drawing $\hat{\mathbf{t}}$, which leads to variation in the numerator, $\tau_{\Delta_{i}}$. Greater distances between a given $\tau$ and $\hat{\mathbf{t}}$ cause $\hat{f}(\tau)$ to be lower for the relevant $\tau$, while smaller distances cause it to be higher. Over the course of the simulation, this variability also leads to different optimal values for $g$, the bandwidth modifier. So during the simulation when $g$ is allowed to vary, the primary source of variability in both $g$ and in $\hat{f}(\tau)$ is variability in $\tau_{\Delta_{i}}$. Since $\tau$ is just an arbitrary fixed position on the time-axis, the variability in $\tau_{\Delta_{i}}$ also reflects variability in the temporal distance between radiocarbon samples around a given $\tau$. Each time $\hat{\mathbf{t}}$ is drawn, the temporal distances between samples in $\hat{\mathbf{t}}$ changes, which causes event-dates to cluster around some $\tau$ and disperse away from others. As the clustering fluctuates around a given $\tau$, the level of the corresponding optimal KDE model at that $\tau$ changes in response. It is these fluctuations in the shape of the optimal $\mathrm{KDE}$ that are captured in the KDE ensemble output by OxCal and combined in a likelihood-weighted average. Thus, the level of the primary KDE model at a given time $(\tau)$ should be interpreted as an estimate of the temporal density of radiocarbon samples weighted by uncertainty about the temporal distance between them.

Importantly, the second part of this interpretation means that a KDE model does not solely reflect through-time changes in the number of radiocarbon samples-it reflects chronological uncertainty as well. Just like the SPDF, changes in the level of the proxy are also a function of chronological uncertainty, not simply the number of radiocarbon samples dated to a particular time. This mixture of through-time variation in the number of dated events and chronological uncertainty is partly what makes the KDE useful as a summary of chronological information for a large databases of radiocarbon dates. It also, however, raises problems for interpreting specific fluctuations in the model. Ups and downs in the level of the curve cannot be directly interpreted as fluctuations in the number of events dated to a given time, nor the temporal density of events at a given time. The fluctuations also represent uncertainty about the temporal locations of the events in question, specifically uncertainty about the temporal distance between them. Consequently, fluctuations in the model cannot be straightforwardly interpreted as directly indicative of through-time fluctuations in the number of events or the corresponding target process. As Ramsey explained, the KDE approach “... can be used to summarize the distribution of events..." in large ${ }^{14} C$ databases (emphasis added) (Ramsey, 2017, p.1831). It is a summary of chronological information, not a simple reflection of through-time variation.

\section{Simulated Regression Experiment}

The most promising potential use for radiocarbon-date proxies, as we mentioned earlier, is understanding the causes and nature of their intended target phenomenon. Explaining through-time variation in a given proxy has a been a core motivation for research involving the proxies since they were originally devised (e.g., Collard et al., 2010; Colledge et al., 2019; Demján and Dreslerová, 2016; Geyh, 1969; Palmisano et al., 2017; Rick, 1987; Shennan, 2009, 2013; 
Shennan et al., 2013). This motivation naturally leads to regression models wherein a given proxy serves as a response variable that is potentially a function of one or more covariates. Therefore, despite the potential biases and interpretive problems, it is important to know whether radiocarbon-date proxies could nonetheless be used as intended in the context of regression modelling. To find an answer, we ran an experiment using simulated data.

\subsection{Materials and Methods}

\subsubsection{Simulated Data}

We created two simulated time-series for the experiment (see Fig. 3). One was a series of "true" calendar dates for a set of radiocarbon samples and the other was a covariate that determined through-time variations in the number of samples. The former served as the analogue to a database of radiocarbon samples, while the latter was an analogue for a causally-relevant process. These variables were defined by several equations.
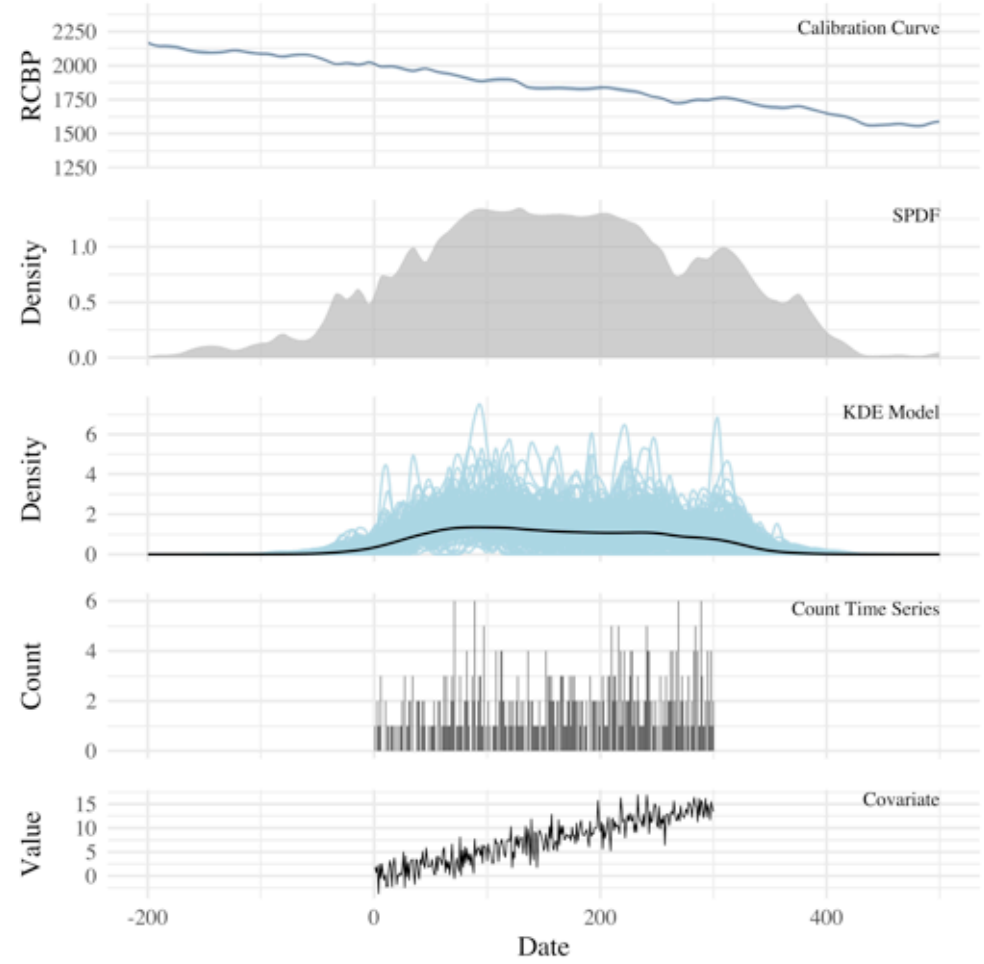

Figure 3: Simulated data and radiocarbon-date proxies. Note that the panel labelled "KDE Model" displays 500 samples of the KDE ensemble as blue, slightly transparent lines while the main likelihood-weighted KDE model is a single black line.

The number of radiocarbon samples dated to a given calendar year were denoted $y_{\tau}$ where $\tau$ represented a single year from 1-300 CE. This span of years was chosen in order to limit the final number of radiocarbon dates to between 400 and 500, which was a trade-off between recommended sample sizes (e.g., Williams, 2012) and computation time. The span also covered approximately the same portion of the INTCAL2013 calibration curve (Reimer et al., 2013) used in 
Ramsey (Ramsey, 2017) to explore the KDE model. Each observation, $y_{\tau}$, was a realization of a Poisson process with a time-varying mean, $\lambda_{\tau}$,

$$
y(\tau) \sim \operatorname{Pois}\left(\lambda_{\tau}\right) .
$$

The Poisson mean, $\lambda_{\tau}$, was a product of the simulated covariate, $x_{\tau}$,

$$
\lambda_{\tau}=e^{x_{\tau}},
$$

and each $x_{\tau}$ was, in turn, a realization of a normally-distributed random variable,

$$
x(\tau) \sim N(0.05 \tau, \sigma) .
$$

We included a simple linear trend in the mean of $x_{\tau}$ with a slope of 0.05 to create a clear increasing signal that produced between 400 and 500 simulated events. Given the linear relationship between $y_{\tau}$ and $e^{x_{\tau}}$, the slope is also the true value for the regression coefficient that would be estimated by a regression model. It, therefore, served as the target value for our experiment.

\subsubsection{Radiocarbon-date Calibration and Proxy Calculations}

The series of counts, $y_{\tau}$ was then reformatted into a set of calendar dates and subsequently fed into the radiocarbon-date calibration program OxCal (Ramsey, 2009). We used OxCal's 'R_Simulate' function to turn the dates into a set of plausible radiocarbon assay results corresponding to the input calendar dates (Ramsey, 2009). For simplicity, we specified a constant error of \pm 50 -years for every date. The simulated radiocarbon dates were then used in two separate calibration models, one in which we used OxCal's 'Sum' command and another in which we used its new 'KDE_Model' command. Each command acted as a 'grouping' for an OxCal calibration model (Ramsey, 2009, 2017). We included no prior constraints on the boundaries of the groupings or on the distribution of dates within the groupings. In a real world case, these would probably be appropriate choices for a regional database of stratigraphically unrelated radiocarbon samples.

Each of the two OxCal commands produced a different type of radiocarbon-date proxy (see Fig. 3). The 'Sum' command, of course, produced the usual summed proxy, which we refer to as an SPDF. The 'KDE_Model' command produced a primary KDE model and an ensemble of KDE models each of which was calculated using a different bandwidth parameter explored during the course of OxCal's MCMC simulation. To reiterate, the primary KDE model 
is a likelihood-weighted average of the ensemble and the ensemble can be used to approximate an uncertainty envelope around the primary model (Ramsey, 2017). We extracted 200 individual KDEs from the ensemble produced by OxCal.

\subsubsection{Regression Models}

Our regression analysis was based on Bayesian models (Gelman et al., 2014) and it involved three steps. In the first step, we sought to confirm that we could reconstruct the relationship between the true (i.e., simulated) response variable and covariate without any chronological uncertainty. To do so, we created a regression model using the true count time-series and covariate. Since the response data represented integer counts produced by a Poisson process, we used a Poisson-based regression model that mirrored eqs. 5.1-5.3. We also included an intercept and set of priors on the regression coefficients. The model was specified as follows,

$$
y(\tau) \sim \operatorname{Pois}\left(e^{\beta_{0}+\beta x_{\tau}}\right),
$$

where $\beta$ and $\beta_{0}$ were the regression coefficients for the covariate $(x)$ and an intercept, respectively. As in all previous equations, $\tau$ represented time. The regression coefficient parameters had the following uninformative priors,

$$
\begin{gathered}
\beta_{0} \sim N(0,1000) \\
\beta \sim N(0,1000) .
\end{gathered}
$$

Then, in the second step, we constructed a regression model for the radiocarbon-date proxies. Both the SPDF and KDE models were continuous and always positive by definition. Thus, we based this model on the Gamma distribution. The regression model was specified as follows,

$$
y(\tau) \sim \Gamma\left(r, \frac{r}{e^{\alpha+\beta x_{\tau}}}\right),
$$

where $\beta$ was again a regression coefficient, and $r$ was the rate parameter of the Gamma distribution. The other parameter, $\alpha$, was an autoregression term. Including an autoregression term in the models for the SPDF and KDE was necessary because they contain significant autocorrelation (see Fig. 4). We modelled this autocorrelation with a normally-distributed, latent autoregressive process. The process was defined as follows,

$$
\alpha_{\tau} \sim N\left(\rho \alpha_{\tau-1}, \sigma\right)
$$


where $\rho$ was an autoregression coefficient and $\sigma$ was the standard deviation of the process. Each $\alpha_{\tau}$ was a product of $\rho$ and the previous $\alpha_{\tau-1}$ with some minor additive noise represented by $\sigma$. We used another latent parameter, $\alpha_{0}$, to represent the level of the autoregressive process in the period before the start of the data. The following priors were then applied to the process parameters,

$$
\begin{aligned}
\sigma & \sim \operatorname{dexp}(0.75), \\
\rho & \sim N(0,10), \\
\alpha_{0} & \sim N(0,10) .
\end{aligned}
$$

It is important to note that $\alpha_{0}$ also acted like an intercept in these models because it estimated the initial level of the autoregressive process.
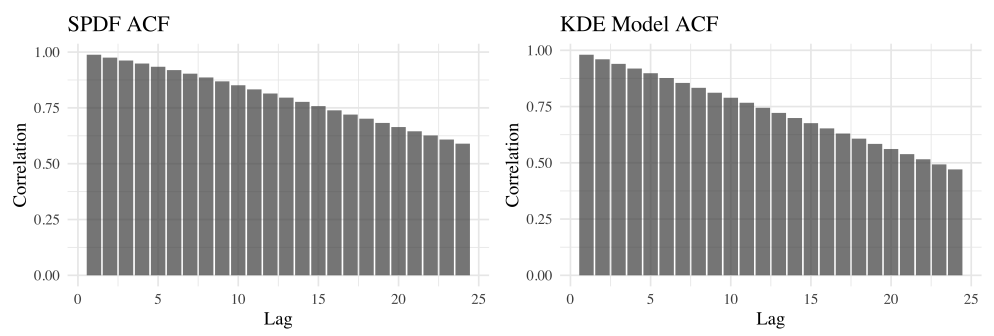

Figure 4: Plots showing autocorrelation present in the SPDF and KDE proxies. The height of each column in the plot corresponds to the correlation between a given proxy and itself at the lag indicated on the x-axis. As the plots show, both proxies are highly autocorrelated.

We also applied uninformative priors for the regression parameters as follows,

$$
\begin{aligned}
& \beta \sim N(0,1000) \\
& r \sim \operatorname{Exp}(0.01) .
\end{aligned}
$$

The Gamma rate, $r$, was a nuisance parameter for which we used a wide exponentially-distributed prior. This prior was chosen because the parameter had to be positive and non-zero given the standard definition of the Gamma distribution. Lastly, we used a hierarchical probability model to analyse the KDE ensemble provided by OxCal. We performed this analysis because the individual KDE models in the ensemble involved different bandwidths, as we explained earlier. So, each model in the ensemble, $\mathbf{K D E}_{\mathbf{E}}=\left\{K D E_{E_{1}}, K D E_{E_{2}}, \ldots, K D E_{E_{n}}\right\}$, represented not only one probable set of dates for the simulated radiocarbon samples but also a different bandwidth. The different bandwidths, in a sense, reflected different temporal scales. When the bandwidth was smaller, the KDE model was more variable-i.e., 
spikier-reflecting variation in the density over shorter timescales. With a larger bandwidth, the KDE represented variation in the density over longer timescales, and it was much smoother. Estimating individual regressions for each $K D E_{E_{i}}$ and then combining the results allowed us to account for uncertainty in the bandwidth parameter. To combine the individual models, we created a Bayesian hierarchical model (Gelman et al., 2014). Each $K D E_{E_{i}}$ regression model was considered a sample of a population of potential regression models. This population shared a set of latent top-level parameters. The grand regression parameter was represented by $\beta$, and the sampling uncertainty was represented by $\sigma$. Each lower-level model was specified as follows,

$$
y_{i}(\tau) \sim \Gamma\left(r_{i}, \frac{r_{i}}{e^{\alpha_{i}+\beta_{i} x_{\tau}}}\right)
$$

We again used largely uninformative priors. These priors were defined as follows for the lower-level models that each involved a different KDE ensemble member,

$$
\begin{aligned}
\beta_{0_{i}} & \sim N\left(\beta_{0}, \sigma\right) \\
\beta_{i} & \sim N(\beta, \sigma) \\
r_{i} & \sim U\left(e^{-7}, 100\right) .
\end{aligned}
$$

The priors for these low-level models had as their means the top-level (i.e., grand) regression parameters, which had priors defined as follows,

$$
\begin{aligned}
& \beta \sim N(0,1000) \\
& \sigma \sim U\left(e^{-7}, 100\right) .
\end{aligned}
$$

In each step of the regression experiment, we employed the same logic for evaluating the results. We reasoned that a given model had adequately reconstructed the true response-covariate relationship if it met two criteria. One is that at least $98 \%$ of the posterior distribution for $\beta$ was greater than zero. The other criterion was that the mean of the posterior should be close to the true value of the regression coefficient, $\beta=0.05$. If only the first criterion was met, a given model was deemed indicative, but biased-i.e., it still reflected an important aspect of the target relationship, namely that the true response and covariate were positively correlated, but the estimate of the strength of the relationship would be wrong. If neither criterion was met, the relevant model was deemed inadequate, having produced a false negative. An indicative-but-biased or inadequate result with respect to the first regression model - the one involving the true response-covariate pair-would imply that the simulated signal was insufficiently strong and that the rest of the 
analysis would not be useful for evaluating the proxies. An indicative-but-biased result with respect to one of the models involving a proxy indicated that the relevant proxy might be useful for regression studies under certain conditions, but that the results of such analyses should be carefully interpreted because of the bias. Lastly, an inadequate result with respect the one of the proxy models indicated that the relevant proxy should not be used in regression analyses.

All of the analyses were conducted in R (R Core Team, 2019). We used a Bayesian analysis package, 'Nimble' (NIMBLE Development Team, 2018), to produce the models and estimate their parameters with MCMC simulations. These simulations each involved tens of thousands of iterations. We visually inspected the MCMC posterior chains for diagnostic indications of convergence problems. We also used standard Geweke statistics (Geweke, 1992) to test the chains for significant deviations from stationarity after discarding the first 1000 iterations of a given chain as burn-in (see Supplementary Information for Geweke test results). The packages 'stringr' (Wickham, 2019), 'ggplot' (Wickham, 2016), and 'ggpubr' (Kassambara, 2019) were used for data processing and producing figures. All of the R scripts used in this analysis are available at www.github.com/wccarleton/sumthings.

\subsubsection{Regression Results}

Step 1: Sanity Check. The first regression analysis revealed that we could reconstruct the true response-covariate relationship in the absence of chronological uncertainty. Figure 5 shows the true response and covariate data along with a scatter plot of the two variables and the posterior distribution for $\beta$ from the first regression model. As the plots illustrate, there is a clear linear relationship between the number of simulated events and variation in the covariate. The model also produced a posterior estimate for the regression coefficient that includes the true value in the $98 \%$ credible interval. This is a true-positive result from an adequate model.
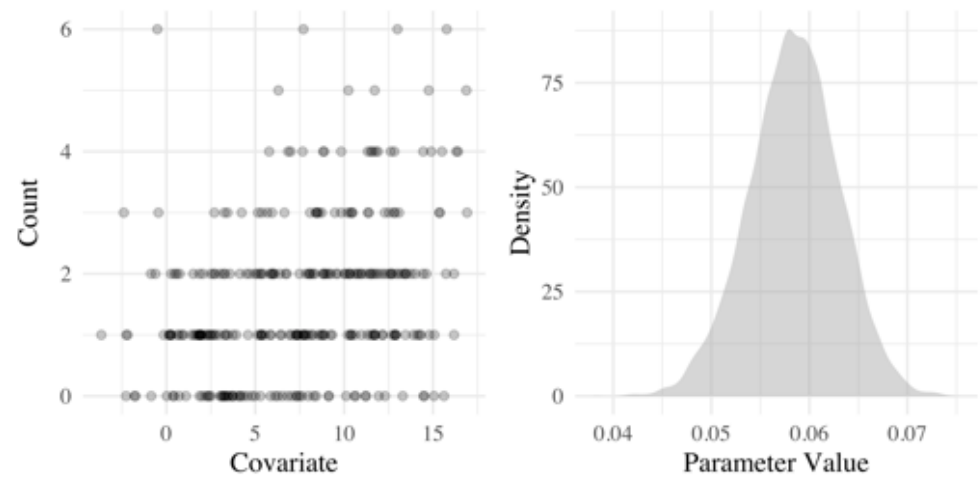

Figure 5: Plots showing the response-covariate relationship for the simulated data and the posterior estimate of the target parameter from the Poisson regression model. The scatter plot (left) shows that there is a clear linear relationship between the simulated covariate and response count data-as would be expected. The posterior distribution (right) is very close to the true target value, indicating that the signal in the simulated data is clear enough to recover in the absence of chronological uncertainty.

Step 2. SPDF and KDE model. The second regression analysis, in contrast, produced two inadequate results. Figure 6 shows the posterior distributions from the regression models involving the SPDF and Fig. 7 shows the results for the primary KDE model. As these plots indicate, the posterior distributions for $\beta$ in both cases include zero within the $98 \%$ 
credible region of each density. According to these results, the strength of the proxy-covariate relationship cannot be distinguished from zero for either the regression involving the SPDF or the one involving the KDE model. These are false-negative findings from two inadequate models.

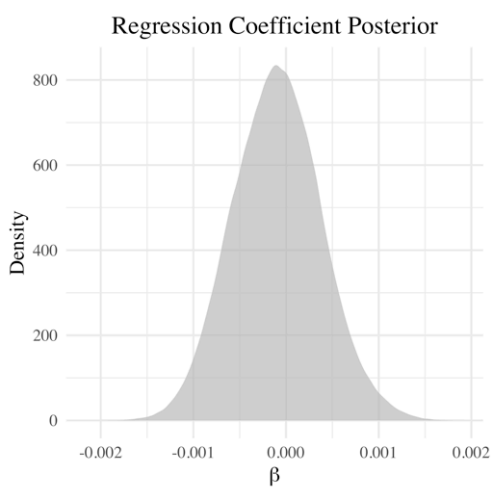

Figure 6: Regression results for the model involving the SPDF proxy. The posterior densities for the main regression parameter is centered on zero, which indicates no relationship was identified. This is a false-negative result.

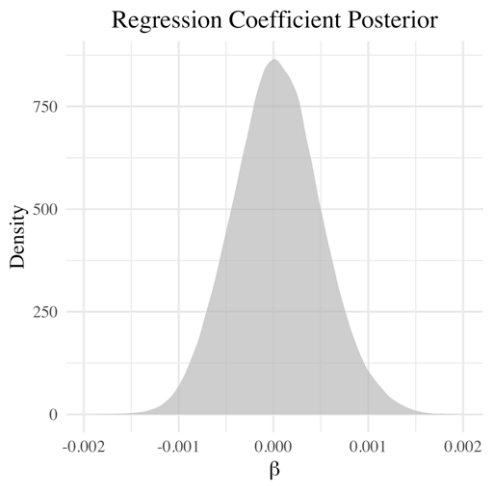

Figure 7: Regression results for the model involving the KDE proxy. The posterior densities for the main regression parameter is centered on zero, which indicates no relationship was identified. This is a false-negative result.

Step 3. KDE Ensemble. Unfortunately, the results of the third regression analysis were similarly disappointing. The posterior distribution for the top-level regression coefficient, $\beta$, is effectively centered on zero (see Fig. 8). This means that, on average, the regression coefficients estimated for the models from the KDE ensemble indicate no relationship between the KDE proxies and the covariate. The overall result from this analysis was also a false negative.

\section{Discussion}

Our two-part analysis revealed a major, underappreciated problem with radiocarbon-date proxies: they do not represent the processes they are often thought to represent. It is important to stress that the problem does not extend to the method of radiocarbon dating more generally —only the use of dates as proxies. This problem was clearly demonstrated in both parts of our analysis. In the first part, we looked carefully at the mathematics defining each proxy. The mathematical definition of the well-known SPDF proxy indicates that it represents variation with respect to time in the probability 


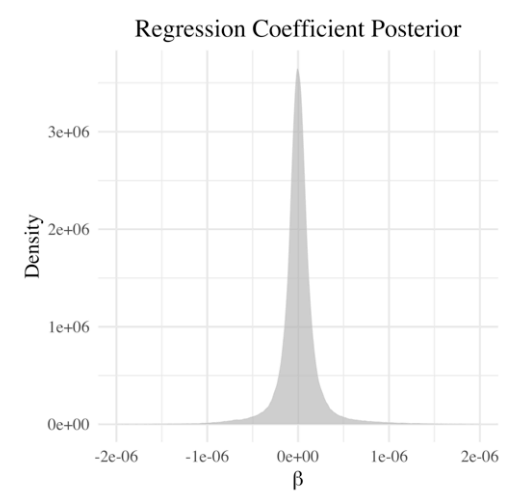

Figure 8: Regression results for the model involving the KDE ensemble. The posterior densities for the top-level main regression parameter is centered on zero, which indicates no relationship was identified on average among the individual, low-level KDE regression models. This is a false-negative result.

that at least one event in a given database occurred at a given time-and, critically, it does not indicate whether any particular number of events occurred. The equations behind the new KDE proxy indicate it represents an estimate of the temporal density of radiocarbon-dated events weighted by uncertainty about the temporal distance between the events. Both proxies conflate chronological uncertainty with through-time variation. Neither, therefore, should be expected to represent through-time changes in any target phenomenon related to the number of radiocarbon samples recovered in palaeoenvironmental or archaeological records. Treating them as if they do ignores the real meaning of the proxies. This suggests that perhaps SPDFs and KDE models should not be used as proxies for through-time processes and they may be unsuitable for analyses that seek to explain through-time variation in a given target process.

The second part of our analysis, the regression experiment, supported this conclusion. It revealed that both proxies can produce false negative results. To reiterate, we simulated a set of radiocarbon-dated events with a single causal covariate, calibrated the resulting dates, and then used the dates to produce SPDF and KDE models. The models were then used as response variables in regression analyses where they served as proxies for the "true" underlying time-series of radiocarbon-dated events. In each regression model, the true covariate was also included. All of our analyses produced false negative results. Neither the SPDF nor the KDE model were adequate proxies for the true underlying simulated process. Regressions involving these proxies all failed to recover the direction or strength of the known association between the target process and its covariate.

During our analyses, we realized there are two additional features of radiocarbon-date proxies that we think will always limit their analytical utility. One involves a special kind of non-independence. In both the SPDF and KDE model, neighboring observations have an unusual effect on each other. The effect is that if one observation is taken to be true-i.e., accurately reflects the underlying number of events at a given time- the neighbouring observations must be false. This is because the events on which a given model is based can only have occurred at one time and not another. To illustrate, consider a single calibrated radiocarbon-date density. Variations in the level of the density indicate the relative probability that the event in question occurred at a given time. If it did occur in a particular time, however, it cannot have occurred in another time. The same logic applies to the SPDF. If one assumes that the level of the 
proxy at time $\tau$, for instance, reflected the number of events at time $\tau$, the number of events at neighbouring times $(\tau \pm 1, \tau \pm 2, \ldots)$ must necessarily be different than the proxy indicates. This is, of course, because events contributing to the probability at time $\tau$ cannot simultaneously contribute to the value at neighboring times. The problem is similar for the KDE model - though, in that case, it is the magnitude of the contribution of a given event to the density at $\tau$ that must change in response to fixing any given event to a particular $\tau$. As a result, radiocarbon-date proxies cannot be mapped to covariate observations in a stable, consistent way. It is impossible to determine whether a given proxy observation — say the level of an SPDF at time $\tau_{2}$ —should be paired with a potential covariate observation at time $\tau_{1}$, $\tau_{2}, \tau_{3}$, or some other time. Importantly, this problem is more than just additional measurement error, and as far as we can tell there is no standard statistical model that properly accounts for this strange non-independence.

The second limiting feature is a property of probability density functions. Any density function has to have a positive finite integral-i.e., the area between a continuous density function (curve) and its domain (x-axis) has to be entirely above the domain (positive everywhere) and it has to have a finite limit (the area cannot be infinitely large, and in the case of valid probability densities it has to be equal to one) (Blitzstein and Hwang, 2015). Put another way, the area between a density curve and its domain has to be fully enclosed by the curve. This means that the curve can go up and down in any manner, but it must "begin" by going up and "end" at some point by going down. This property is true of uncalibrated radiocarbon date densities and calibrated date densities. By extension it is also true of an SPDF and by definition it is true of the KDE model. This means that any SPDF and/or KDE model will follow a similar pattern with respect to time- the curves will generally increase and then decrease over some interval of time. Sometimes, a given proxy may exhibit several up/down fluctuations, but overall the up-then-down pattern will be consistent irrespective of the true through-time structure of the target process. Consequently, whatever the true through-time relationship looks like for a given process-covariate pair, the relationship between a corresponding radiocarbon-date proxy and the covariate will be distorted. This was particularly clear in our simulated regression experiment. The true series of events was linearly related to the simulated covariate, but the proxies had a curvilinear relationship with the covariate (see Fig. 9). It is important to recognize that this is not a distortion that can be corrected-it is a feature of the proxies that they do not generally have the same relationship to a given covariate that the target process does. This is precisely because the proxies do not actually reflect the target process, which means regressions involving SPDF or KDE models are likely to produce misleading results. It is equally important to recognize that this problem would be very difficult to identify in a real-world case study because many potential covariates will not be simple linear functions of time. This means that real potential covariates may happen, by chance, to have up-then-down patterns similar to those of radiocarbon-date proxies leading to spurious correlations.

Our findings have important implications for past and future research. A major implication for previous research is that published findings based on radiocarbon-date proxies could well be misleading - this may apply to hundreds of individual papers, including many published in the highest profile journals. In more qualitative studies involving no regression models (e.g., Bradtmöller et al., 2012), narratives about through-time processes like demographic changes or fluctuations in sea level are probably telling the wrong story. In more quantitative research involving correlation and/or 

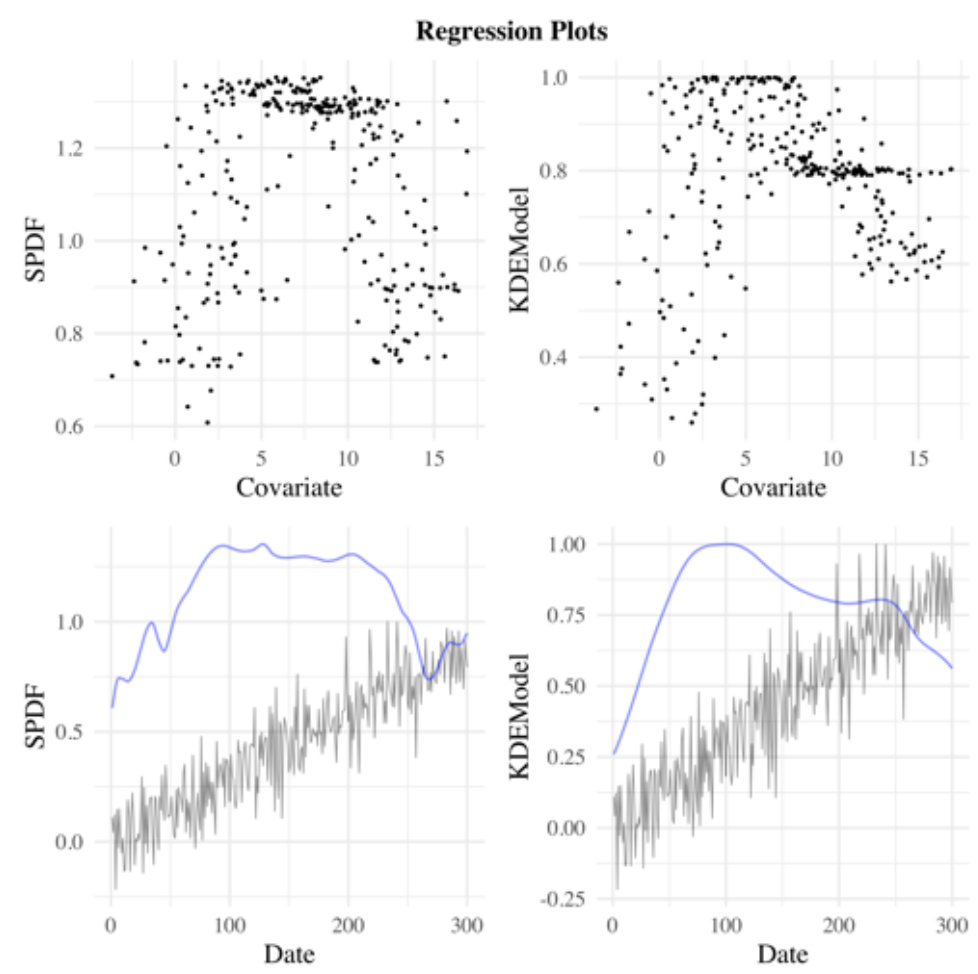

Figure 9: Plots illustrating the curvilinear relationship between radiocarbon-date density proxies and the simulated covariate. The top two plots are scatter plots comparing the SPDF (left) and KDE model (right) to the simulated covariate. The bottom two plots are showing the same data, but in the time domain (blue lines represent the radiocarbon-date proxies).

regression (e.g., Ebert et al., 2017), hypothesis tests could be invalid and parameter estimates false or biased, calling into question any interpretations and conclusions drawn on the basis of such findings. Many of these published studies should be carefully re-evaluated to determine whether the problems we identified undermine previous conclusions. We strongly suspect that they will.

A major implication for future research is that these proxies should be avoided when the research goal is understanding through-time variation in a given process. The proxies represent distributions of chronological uncertainty with respect to time, as we have stated. So, analyses intended to understand how/why chronological uncertainty fluctuates with respect to time could, for instance, profitably use the proxies as response variables in standard regression models. The proxies may also be of use for addressing questions about chronological relationships. Research aimed at, for example, determining whether a collection of events pre- or post-dates a fixed date could also be based on the established proxies-e.g., the case study in Ramsey's KDE model paper 2017. Research intended to improve our understanding of through-time fluctuations in the number of radiocarbon-dated events, however, should not be based on the proxies. Researchers can no longer assume that radiocarbon-date proxies represent through-time variation in processes that led to the deposition of organic radiocarbon in the archaeological or palaeoenvironmental records.

We imagine future research proceeding in two main directions. One involves further statistical research like the regression experiment we conducted. It would be very useful to know more about the relationship between the 
parameters we used to simulate the data and the statistical power of regression models in which a given proxy is used as a response variable. We selected parameters that we think created a strong, identifiable signal and simulated a dataset that is reasonably characteristic of the kinds used in research so far-i.e., a simple increase in the number of events, a time-span of a few centuries, hundreds of individual dates, etc. Changing some of these parameters and rerunning the experiment could help identify conditions under which the proxies give better or worse results. Permuting combinations of parameters and rerunning the experiment multiple times would also allow researchers to estimate accurate true and false positive rates for each proxy under various experimental conditions. While this research is unlikely to contradict our main findings, it may help researchers to fully understand the scale of the problem.

The other direction involves methodological research aimed at developing better proxies. While the two established methods for creating radiocarbon-based proxies — the SPDF and KDE model—appear to be unsuitable for their intended purpose, it is possible that radiocarbon date databases could be used to create another proxy, one that does reflect through-time variation in the number of radiocarbon-dated events (e.g., Baxter and Cool, 2016). In this regard, high-precision radiocarbon-dating methods could be very important and this possibility should certainly be explored. Other dating methods_e.g., Uranium/Thorium, Potassium/Argon, etc.-may make better proxies, and so should be investigated as well. It would, however, be advisable to avoid too myopic a focus on dates-as-data approaches and, instead, divert some effort toward developing and exploring proxies based on other data types altogether. Both the archaeological and palaeoenvironmental records contain vast and various data types. In light of the rather bleak outlook for radiocarbon-based proxies, the potential for building replacement proxies based on these other data types also needs to be investigated.

\section{Conclusion}

Radiocarbon-date proxies are tempting to use and have appeared in hundreds of scholarly articles since their inception. They are intended to represent through-time fluctuations in the amount of radiocarbon in the archaeological and palaeoenvironmental records. These through-time fluctuations are thought to be caused by variation in one or more radiocarbon-producing target processes, including past human activity, sea-level changes, and surging fire regimes. However, there are lurking problems that make radiocarbon-date proxies unsuitable representations of their targets. The main problem is that the proxies do not clearly reflect through-time variation in the number of radiocarbon-dated events in a given database. Rather they combine through-time variation with chronological uncertainty. Unfortunately, this conflation often goes unnoticed leading to faulty interpretations and misleading results. We, therefore, urge scholars to think carefully about whether the proxies are actually indicating what they are thought to be indicating. In many instances, we suspect, they are not. 


\section{Data Availability}

Data used in this article was simulated in an R environment and can be replicated by following using the equations provided above. It can also be replicated in $\mathrm{R}$ by running the relevant scripts contained in the online repository at www.github.com/wccarleton/sumthings.

\section{Acknowledgements}

We thank the Max Planck Society for providing the funding necessary to carry out this research.

\section{References}

Armit, I., G. T. Swindles, and K. Becker

2013. From dates to demography in later prehistoric Ireland? Experimental approaches to the meta-analysis of large 14C data-sets. Journal of Archaeological Science, 40(1):433-438.

Bamforth, D. B. and B. Grund

2012. Radiocarbon calibration curves, summed probability distributions, and early Paleoindian population trends in North America. Journal of Archaeological Science, 39(6):1768-1774.

Baxter, M. J. and H. E. M. Cool

2016. Reinventing the wheel? Modelling temporal uncertainty with applications to brooch distributions in Roman Britain. Journal of Archaeological Science, 66:120-127.

Bleicher, $\mathrm{N}$.

2013. Summed radiocarbon probability density functions cannot prove solar forcing of Central European lake-level changes. Holocene, 23(5):755-765.

Blitzstein, J. K. and J. Hwang

2015. Introduction to Probability: Texts in Statistical Science. Boca Raton, FL: CRC Press.

Bradtmöller, M., A. Pastoors, B. Weninger, and G.-C. Weniger

2012. The repeated replacement model - Rapid climate change and population dynamics in Late Pleistocene Europe. Quaternary International, 247:38-49.

Brown, W. A.

2015. Through a filter, darkly: Population size estimation, systematic error, and random error in radiocarbonsupported demographic temporal frequency analysis. Journal of Archaeological Science, 53:133-147.

Buck, C. E., W. G. Cavanagh, and C. D. Litton

1996. Bayesian approach to interpreting archaeological data. Chichester, UK: John Wiley and Sons, Inc. 
A PREPRINT - NOVEMBER 11, 2019

Buck, C. E., C. D. Litton, and A. F. M. Smith

1992. Calibration of radiocarbon results pertaining to related archaeological events. Journal of Archaeological Science, 19(5):497-512.

Chiverrell, R. C., V. R. Thorndycraft, and T. O. Hoffmann

2011. Cumulative probability functions and their role in evaluating the chronology of geomorphological events during the Holocene. Journal of Quaternary Science, 26(1):76-85.

Collard, M., B. Buchanan, M. J. Hamilton, and M. J. O’Brien

2010. Spatiotemporal dynamics of the Clovis-Folsom transition. Journal of Archaeological Science, 37(10):25132519.

Colledge, S., J. Conolly, E. Crema, and S. Shennan

2019. Neolithic population crash in northwest Europe associated with agricultural crisis. Quaternary Research, Pp. 1-22.

Crema, E. R., A. Bevan, and S. Shennan

2017. Spatio-temporal approaches to archaeological radiocarbon dates. Journal of Archaeological Science, 87:1-9.

d'Alpoim Guedes, J. A., S. A. Crabtree, R. K. Bocinsky, and T. A. Kohler

2016. Twenty-first century approaches to ancient problems: Climate and society. Proceedings of the National Academy of Sciences, 113(51):14483-14491.

Demján, P. and D. Dreslerová

2016. Modelling distribution of archaeological settlement evidence based on heterogeneous spatial and temporal data. Journal of Archaeological Science, 69:100-109.

Devièse, T., I. Karavanić, D. Comeskey, C. Kubiak, P. Korlević, M. Hajdinjak, S. Radović, M. Buckley, S. Pääbo, and T. Higham

2017. New single amino acid radiocarbon dating and DNA analysis of the Vindija Cave Neanderthals. In Proceedings of the European Society for the study of Human Evolution Vol. 6, P. 49.

Ebert, C. E., N. Peniche May, B. J. Culleton, J. J. Awe, D. J. Kennett, N. P. May, B. J. Culleton, J. J. Awe, and D. J. Kennett

2017. Regional response to drought during the formation and decline of Preclassic Maya societies. Quaternary Science Reviews, 173:211-235.

Edinborough, K., M. Porčić, A. Martindale, T. J. Brown, K. Supernant, and K. M. Ames

2017. Radiocarbon test for demographic events in written and oral history. Proceedings of the National Academy of Sciences, 114(47):12436-12441.

Faulkner, P.

2011. Late Holocene mollusc exploitation and changing near-shore environments: a case study from the coastal margin of Blue Mud Bay, northern Australia. Environmental Archaeology, 16(2):173-180. 
A PREPRINT - NOVEMBER 11, 2019

Gamble, C., W. Davies, P. Pettitt, L. Hazelwood, and M. Richards

2005. The archaeological and genetic foundations of the European population during the Late Glacial: Implications for 'agricultural thinking'. Cambridge Archaeological Journal, 15(2):193-223.

García Puchol, O., A. Diez Castillo, and S. Pardo-Gordó

2018. New insights into the neolithisation process in southwest Europe according to spatial density analysis from calibrated radiocarbon dates. Archaeological and Anthropological Sciences, 10(7):1807-1820.

Gayo, E. M., C. Latorre, and C. M. Santoro

2015. Timing of occupation and regional settlement patterns revealed by time-series analyses of an archaeological radiocarbon database for the South-Central Andes ( $\left(16^{\circ}-25^{\circ} \mathrm{S}\right)$. Quaternary International, 356:4-14.

Gelman, A., J. B. Carlin, H. S. Stern, D. B. Dunson, A. Vehtari, and D. B. Rubin

2014. Bayesian Data Analysis, 3rd edition. Boca Raton: CRC Press.

Geweke, J.

1992. Evaluating the Accuracy of Sampling-Based Approachesto the Calculation of Posterior Moments. In Bayesian Stastistics, J. Bernardo, J. Berger, A. Dawid, and A. Smith, eds., Pp. 169-193. Oxford: Clarendon Press.

Geyh, M.

1969. Versuch einer chronologischen Gliederung des marinen Holozäns an der Nordseeküste mit Hilfe der statistischen Auswertung von 14C-Daten. Zeitschrift der Deutschen Geologischen Gesellschaft, 118(January 1969):351-360.

Geyh, M. A.

1971. Middle and young Holocene sea-level changes as global contemporary events. Geologiska Föreningen $i$ Stockholm Förhandlingar, 93(4):679-692.

Geyh, M. A.

1980. Holocene Sea-Level History: Case Study of the Statistical Evaluation of 14C Dates. Radiocarbon, 22(3):695704.

Hoggarth, J. A., S. F. Breitenbach, B. J. Culleton, C. E. Ebert, M. A. Masson, and D. J. Kennett

2016. The political collapse of Chichén Itzá in climatic and cultural context. Global and Planetary Change, 138:25-42.

Kassambara, A.

2019. ggpubr: 'ggplot2' Based Publication Ready Plots. R package version 0.2.2.

Kerr, T. R. and F. McCormick

2014. Statistics, sunspots and settlement: Influences on sum of probability curves. Journal of Archaeological Science, 41:493-501.

Leipe, C., T. Long, E. A. Sergusheva, M. Wagner, and P. E. Tarasov 2019. Discontinuous spread of millet agriculture in eastern Asia and prehistoric population dynamics. Science Advances, 5(9):eaax6225. 
Lepofsky, D., K. Lertzman, D. Hallett, and R. Mathewes

2005. Climate Change and Culture Change on the Southern Coast of British Columbia 2400-1200 B. P. : An Hypothesis. American Antiquity, 70(2):267-293.

Manning, K. and A. Timpson

2014. The demographic response to holocene climate change in the Sahara. Quaternary Science Reviews, 101:28-35.

Mooney, S. D., S. P. Harrison, P. J. Bartlein, A. L. Daniau, J. Stevenson, K. C. Brownlie, S. Buckman, M. Cupper, J. Luly, M. Black, E. Colhoun, D. D’Costa, J. Dodson, S. Haberle, G. S. Hope, P. Kershaw, C. Kenyon, M. McKenzie, and N. Williams

2011. Late Quaternary fire regimes of Australasia. Quaternary Science Reviews, 30(1-2):28-46.

NIMBLE Development Team

2018. Nimble user manual. $R$ package manual version 0.6-12.

Palmisano, A., A. Bevan, and S. Shennan

2017. Comparing archaeological proxies for long-term population patterns: An example from central Italy. Journal of Archaeological Science, 87:59-72.

Pierce, J. L., G. A. Meyer, and A. J. Timothy Jull

2004. Fire-induced erosion and millennial-scale climate change in northern ponderosa pine forests. Nature, 432(7013):87-90.

Prentiss, A. M., H. S. Cail, and L. M. Smith

2014. At the Malthusian ceiling: Subsistence and inequality at Bridge River, British Columbia. Journal of Anthropological Archaeology, 33(1):34-48.

$\mathrm{R}$ Core Team

2019. R: A Language and Environment for Statistical Computing.

Ramsey, C.

2008. Radiocarbon dating: Revolutions in understanding. Archaeometry, 50(2):249-275.

Ramsey, C.

2009. Bayesian Analysis of Radiocarbon Dates. Radiocarbon, 51(1):337-360.

Ramsey, C. B.

2017. Methods for Summarizing Radiocarbon Datasets. Radiocarbon, 59(6):1809-1833.

Reimer, P. J., E. Bard, A. Bayliss, J. W. Beck, P. G. Blackwell, C. Bronk Ramsey, C. E. Buck, H. Cheng, R. L. Edwards, M. Friedrich, P. M. Grootes, T. P. Guilderson, H. Haflidason, I. Hajdas, C. Hatté, T. J. Heaton, D. L. Hoffmann, A. G. Hogg, K. A. Hughen, K. F. Kaiser, B. Kromer, S. W. Manning, M. Niu, R. W. Reimer, D. A. Richards, E. M. Scott, J. R. Southon, R. A. Staff, C. S. M. Turney, and J. van der Plicht 2013. IntCal13 and Marine13 Radiocarbon Age Calibration Curves 0-50,000 Years cal BP. Radiocarbon, 55(4):18691887. 
Rick, J. W.

1987. Dates as Data: An Examination of the Peruvian Preceramic Radiocarbon Record. American Antiquity, 52(1):55-73.

Riede, F.

2009. Climate and demography in early prehistory: using calibrated (14)C dates as population proxies. Human Biology, 81(2-3):309-337.

Schiffer, M. B.

1986. Radiocarbon dating and the "old wood" problem: The case of the Hohokam chronology. Journal of Archaeological Science, 13(1):13-30.

Schulting, R.

2010. Holocene environmental change and the Mesolithic-Neolithic transition in north-west Europe: revisiting two models. Environmental Archaeology, 15(2):160-172.

Shennan, S.

2009. Evolutionary demography and the population history of the european early neolithic. Human Biology, 81(2-3):339-355.

Shennan, S.

2013. Demographic Continuities and Discontinuities in Neolithic Europe: Evidence, Methods and Implications. Journal of Archaeological Method and Theory, 20(2):300-311.

Shennan, S., S. S. Downey, A. Timpson, K. Edinborough, S. Colledge, T. Kerig, K. Manning, and M. G. Thomas

2013. Regional population collapse followed initial agriculture booms in mid-Holocene Europe. Nature communications, 4(1):2486.

Silverman, B. W.

1986. Density estimation for statistics and data analysis. London: Chapman and Hall.

Steele, J.

2010. Radiocarbon dates as data: Quantitative strategies for estimating colonization front speeds and event densities. Journal of Archaeological Science, 37(8):2017-2030.

Surovell, T. A. and P. J. Brantingham

2007. A note on the use of temporal frequency distributions in studies of prehistoric demography. Journal of Archaeological Science, 34(11):1868-1877.

Surovell, T. A., J. Byrd Finley, G. M. Smith, P. J. Brantingham, and R. Kelly

2009. Correcting temporal frequency distributions for taphonomic bias. Journal of Archaeological Science, 36(8):1715-1724. 
Taylor, R. E., O. Bar-Yosef, and C. Renfrew

2014. Radiocarbon Dating, Second Edition: An Archaeological Perspective, 2nd edition. Albuquerque, US: Routledge.

Thorndycraft, V. R. and G. Benito

2006. The Holocene fluvial chronology of Spain: Evidence from a newly compiled radiocarbon database. Quaternary Science Reviews, 25(3-4):223-234.

Turney, C. S. and H. Brown

2007. Catastrophic early Holocene sea level rise, human migration and the Neolithic transition in Europe. Quaternary Science Reviews, 26(17-18):2036-2041.

Wickham, H.

2016. ggplot2: Elegant Graphics for Data Analysis. Springer-Verlag New York.

Wickham, H.

2019. stringr: Simple, Consistent Wrappers for Common String Operations. R package version 1.4.0.

Williams, A. N.

2012. The use of summed radiocarbon probability distributions in archaeology: a review of methods. Journal of Archaeological Science, 39(3):578-589. 


\section{SUPPLEMENTARY INFORMATION FOR "SUM THINGS ARE NOT \\ WHAT THEY SEEM: PROBLEMS WITH THE INTERPRETATION AND ANALYSIS OF RADIOCARBON-DATE PROXIES"}

\section{A PREPRINT}

\author{
W. Christopher Carleton \\ Extreme Events Research Group \\ Max Planck Institute for Chemical Ecology \\ 07745 Jena, Germany \\ wcarleton@ice.mpg.de
}

\author{
Huw S. Groucutt \\ Extreme Events Research Group \\ Max Planck Institute for Chemical Ecology \\ 07745 Jena, Germany \\ hgroucutt@ice.mpg.de
}

November 11, 2019

\section{MCMC Diagnostics}

\subsection{Visual Inspection}

With any MCMC-based Bayesian analysis it is important to ensure that the posterior distributions have been appropriately sampled. To determine that this was the case for our MCMC simulations, we visually inspection MCMC chains and applied a standard diagnostic statistic. For the visual inspection, we plotted the MCMC chains (less a burn-in comprising the first 50,000 iterations) for each main parameter of each model (see Figs 1-4) and we generated pairs plots for each model showing the relationship between model parameters (see Figs 5-8). It should be noted that we performed this inspection on all of the lower-level models in the KDE hierarchical regression as well, but since there were 100 models we did not include all of the relevant plots here. All of the plots indicated that the MCMC has produced satisfactory samples of all posterior distributions.

\subsection{Geweke Tests}

The standard diagnostic statistic we employed was the Geweke test (Geweke, 1992). The test is essentially a t-test that compares the distribution of posterior MCMC samples from the first $10 \%$ of a given MCMC chain to the last $50 \%$ of the same chain. If the test finds no significant difference between the means of those distributions, then the MCMC algorithm is considered to have produced a long-run stationary sample of the relevant marginal posterior distribution. 


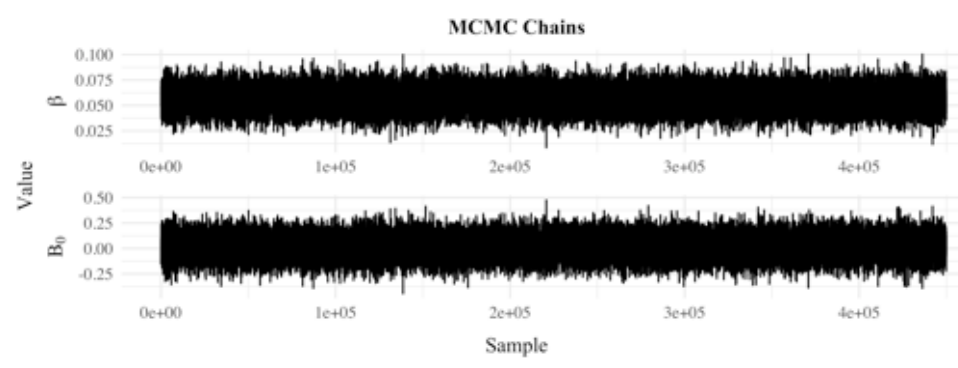

Figure 1: MCMC Chains for parameters of the "Sanity Check" Poisson regression.

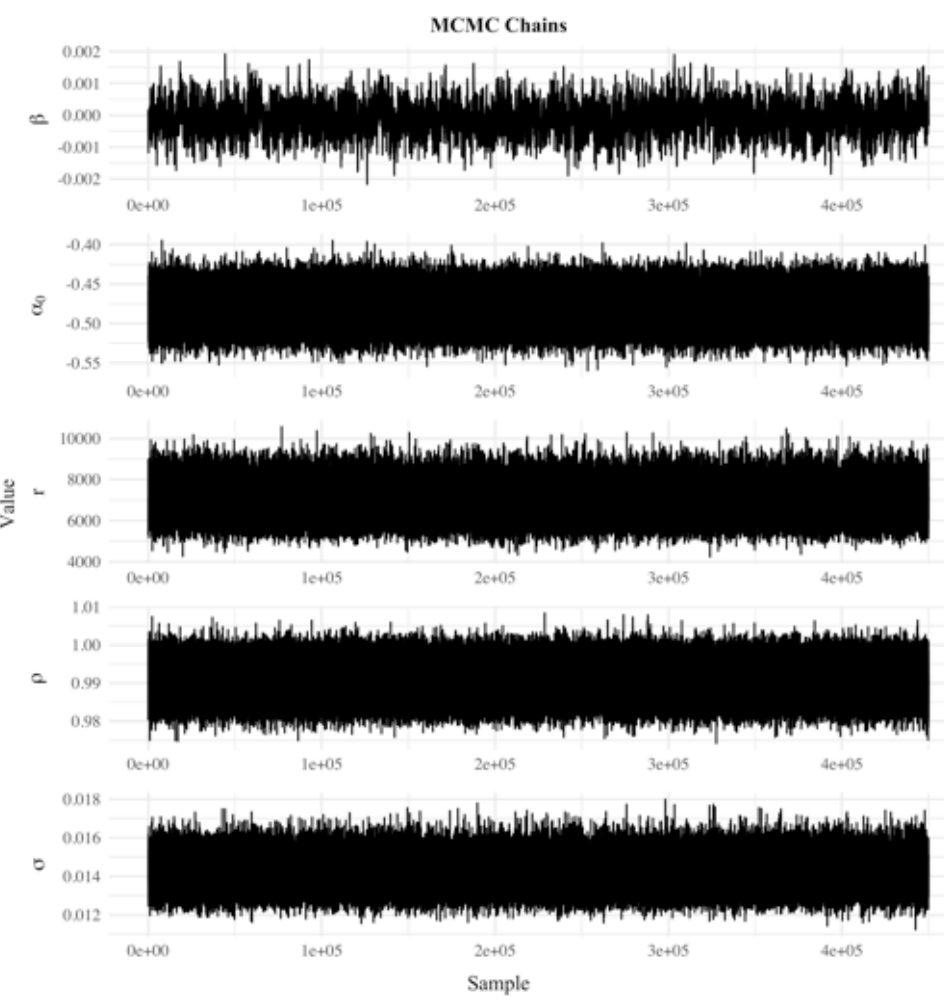

Figure 2: MCMC Chains for parameters of the SPDF regression.

A stationary MCMC chain—often referred to as having "converged"-represents an unbiased sample of the relevant posterior. The following table shows the Geweke statistics for the MCMC chains of the parameters for each of the three models we evaluated. The statistics indicate that the MCMC algorithm converged to unbiased samples of the posteriors in each case, further supporting the visual appearance of convergence in the relevant MCMC trace plots.

\section{References}

Geweke, J.

1992. Evaluating the Accuracy of Sampling-Based Approachesto the Calculation of Posterior Moments. In Bayesian Stastistics, J. Bernardo, J. Berger, A. Dawid, and A. Smith, eds., Pp. 169-193. Oxford: Clarendon Press. 


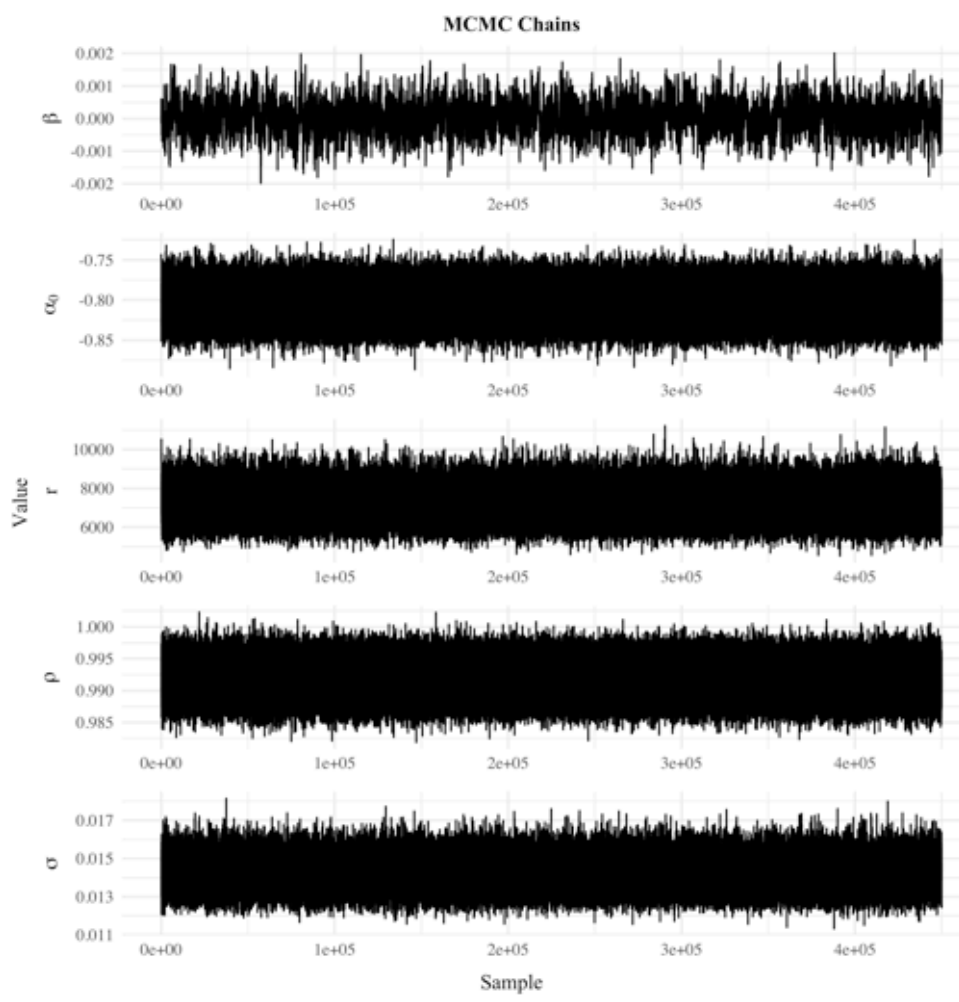

Figure 3: MCMC Chains for parameters of the KDE Model regression.

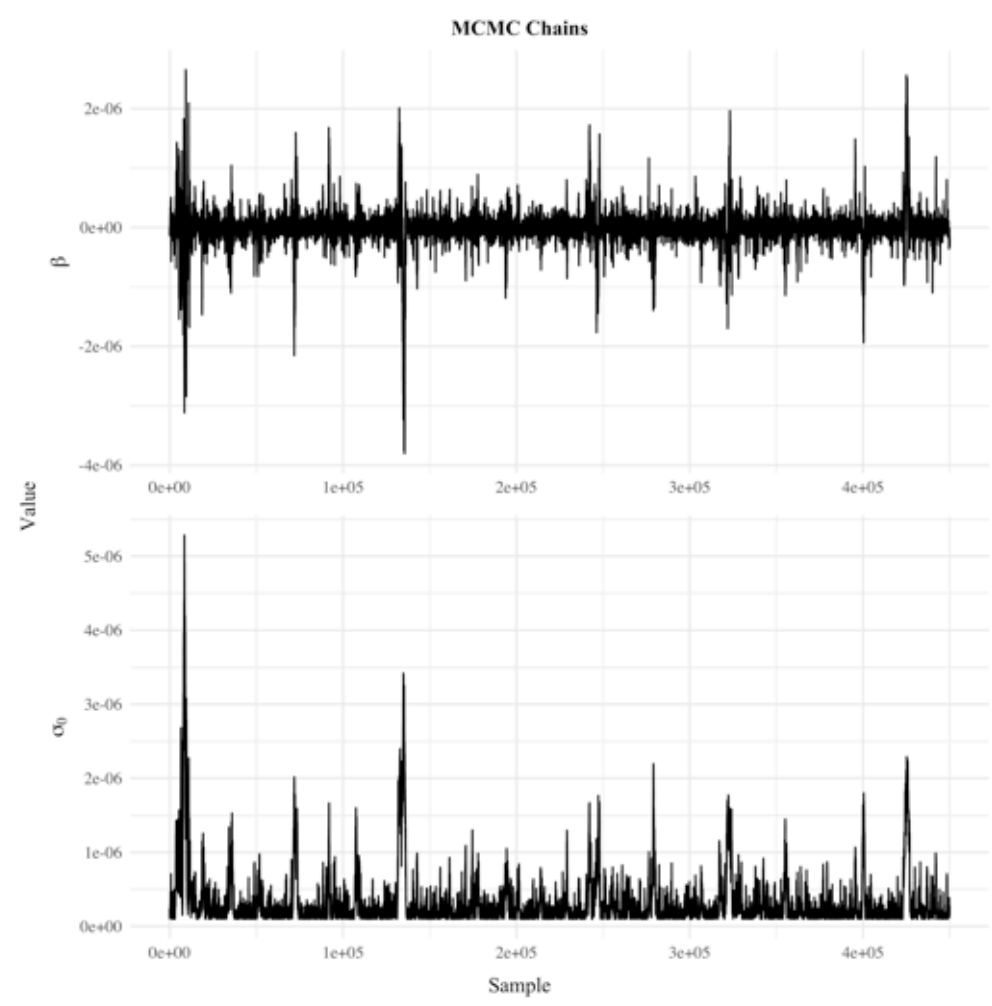

Figure 4: MCMC Chains for top-level parameters of the KDE Model ensemble hierarchical regression. 


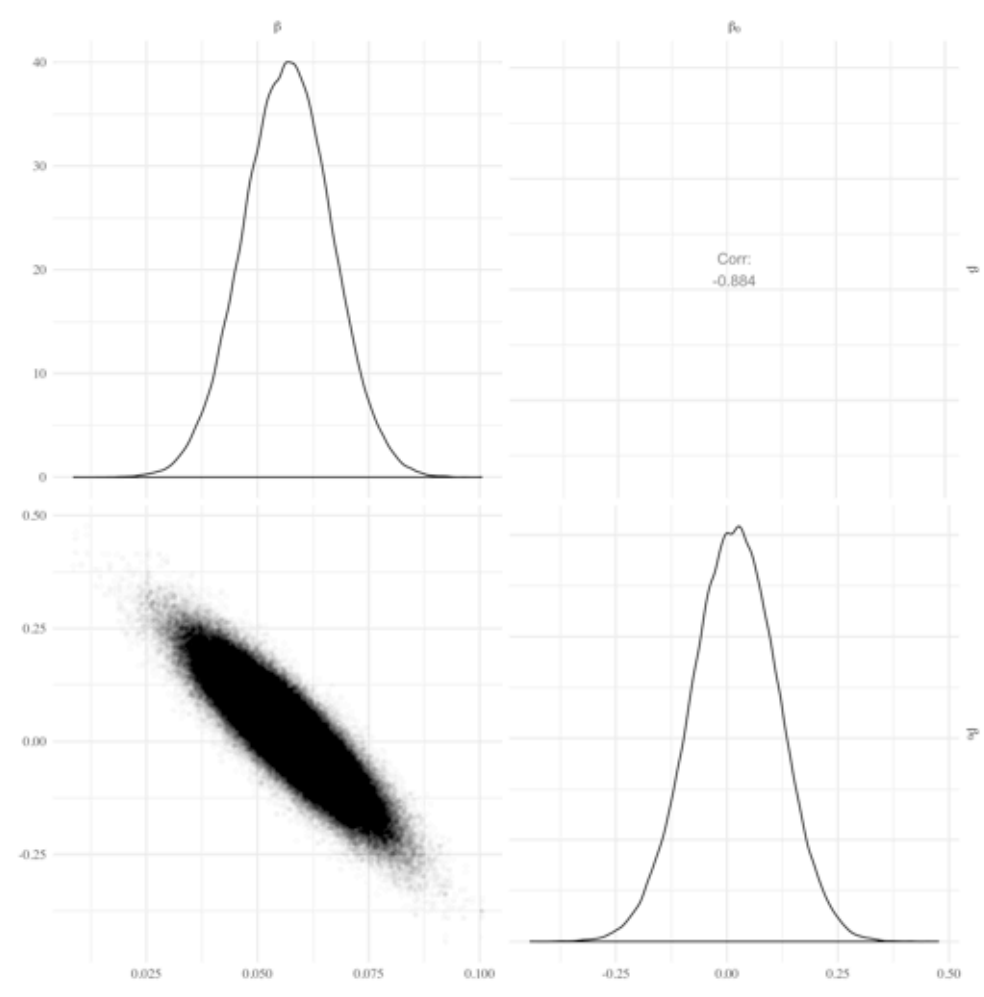

Figure 5: MCMC pairs plot for parameters of the "sanity check" regression in which the simulated count series was used as the response variable.

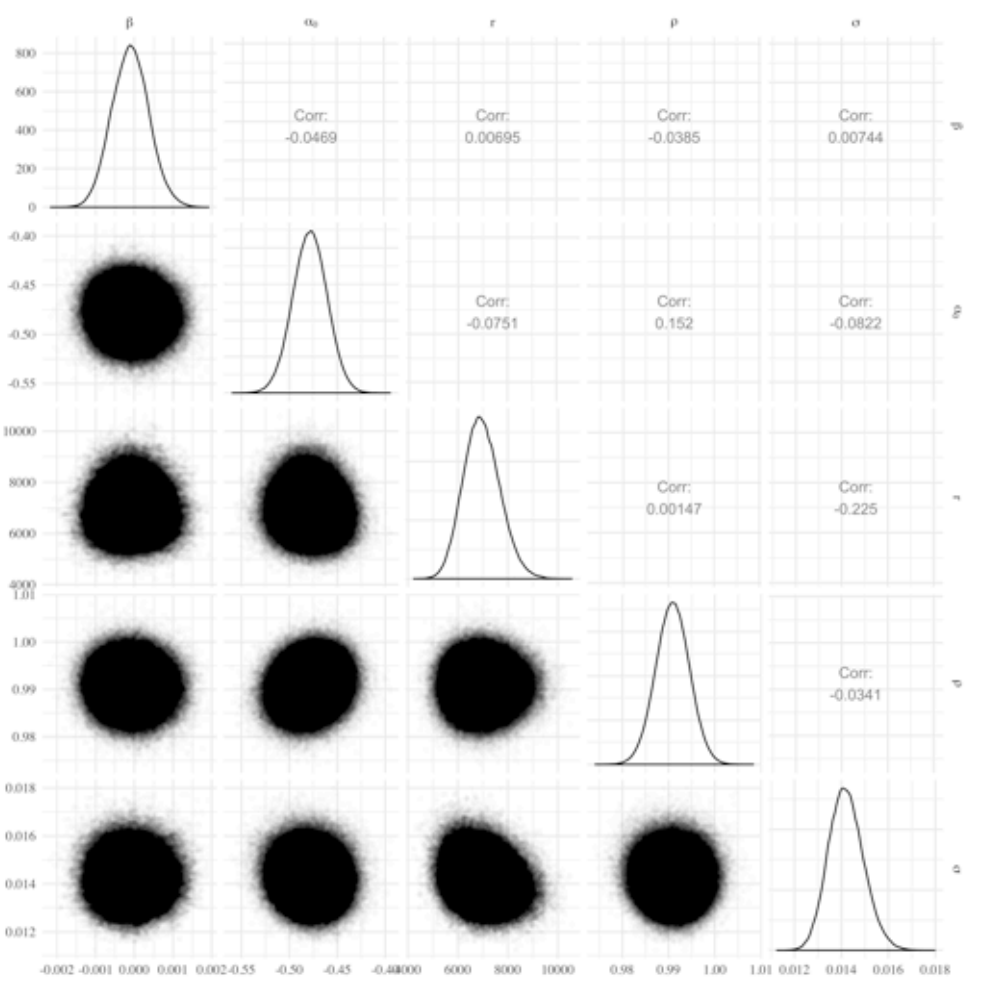

Figure 6: MCMC pairs plot for parameters of the SPDF regression. 


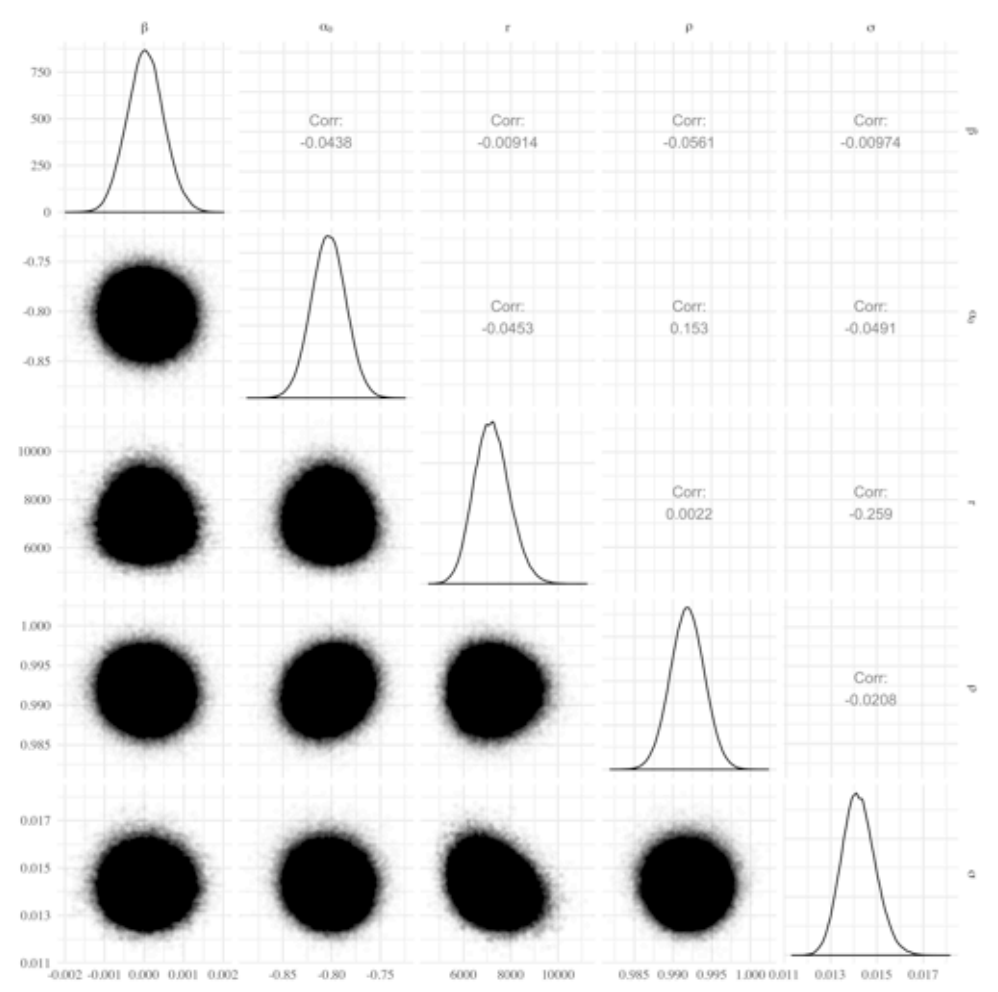

Figure 7: MCMC pairs plot for parameters of the KDE regression.

Table 1: Geweke statistics for MCMC chains of the parameters for the models we analyzed. Each row contains statistics for a given model while the columns contain statistics for a given parameter MCMC chain. The statistics are reported in standard deviations of the standard normal distribution (rounded to the second decimal place), which means that only values larger in magnitude than approximately 2.5 would fall outside the $99 \%$ confidence interval. Since none of the values do, we concluded the MCMC chains were stationary for the parameters in each model, which in turn led us to believe the relevant posterior distributions were appropriately sampled. It should be noted that for reasons of practical space limitations we only report here the Geweke test results for the top-level parameters in the hierarchical model of the KDE ensemble. Corresponding values for the lower-level model parameters can be found in a CSV supplementary file associated with the main manuscript.

\begin{tabular}{llllllll} 
Response & $\beta_{0}$ & $\beta$ & $\alpha_{0}$ & $\mathrm{r}$ & $\rho$ & $\sigma$ & $\sigma_{b}$ \\
\hline Count Series & 1.57 & -1.37 & - & - & - & - & - \\
SPDF & - & -0.62 & -1.57 & 0.73 & 0.24 & -0.95 & - \\
KDE & - & 0.06 & 0.7 & 1.73 & 0.74 & -0.42 & - \\
KDE Ensemble & - & 0.43 & - & - & - & - & -0.15 \\
\hline
\end{tabular}


A PREPRINT - NOVEMBER 11, 2019

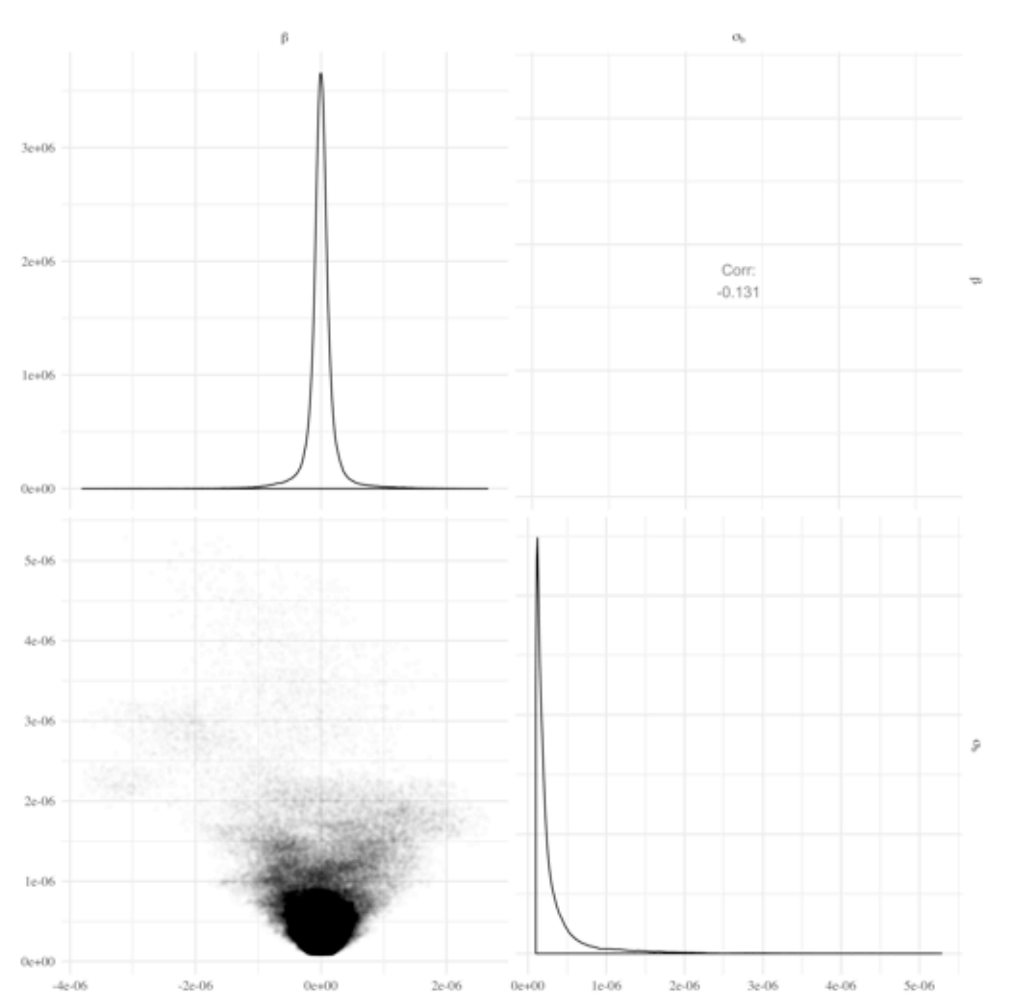

Figure 8: MCMC pairs plot for the top-level parameters of the KDE Model ensemble regression. Practical space limitations meant that we could not present the chains from all lower-level regression models. The slight funnelling of the pairs plot in this image is explained by the role of $\sigma_{b}$ in the hierarchical model. That parameter represents the sampling uncertainty for the regression coefficient $\beta$. A higher sampling uncertainty would lead to greater variability in $\beta$. 